\title{
On large theta-characteristics with prescribed vanishing
}

\author{
Edoardo Ballico ${ }^{1}$. Francesco Bastianelli ${ }^{2}$ (D) Luca Benzo $^{3}$
}

Received: 2 January 2018 / Accepted: 27 October 2018 / Published online: 8 November 2018 (c) Fondazione Annali di Matematica Pura ed Applicata and Springer-Verlag GmbH Germany, part of Springer Nature 2018

\begin{abstract}
Let $C$ be a smooth projective curve of genus $g \geq 2$. Fix an integer $r \geq 0$, and let $\underline{k}=$ $\left(k_{1}, \ldots, k_{n}\right)$ be a sequence of positive integers with $\sum_{i=1}^{n} k_{i}=g-1$. In this paper, we study $n$-pointed curves $\left(C, p_{1}, \ldots, p_{n}\right)$ such that the line bundle $L:=O_{C}\left(\sum_{i=1}^{n} k_{i} p_{i}\right)$ is a theta-characteristic with $h^{0}(C, L) \geq r+1$ and $h^{0}(C, L) \equiv r+1(\bmod 2)$. We prove that they describe a sublocus $\mathcal{G}_{g}^{r}(\underline{k})$ of $\mathcal{M}_{g, n}$ having codimension at most $g-1+\frac{r(r-1)}{2}$. Moreover, for any $r \geq 0, \underline{k}$ as above, and $g$ greater than an explicit integer $g(r)$ depending on $r$, we present irreducible components of $\mathcal{G}_{g}^{r}(\underline{k})$ attaining the maximal codimension in $\mathcal{M}_{g, n}$, so that the bound turns out to be sharp.
\end{abstract}

Keywords Theta-characteristic $\cdot$ Moduli of curves $\cdot$ Spin curve $\cdot$ Subcanonical point

Mathematics Subject Classification $14 \mathrm{H} 10 \cdot 14 \mathrm{H} 45 \cdot 14 \mathrm{H} 51$

This work was partially supported by the national projects FIRB 2012 "Spazi di moduli e applicazioni" and PRIN 2010-11 “Geometry of algebraic varieties" founded by the Ministero dell'Istruzione, dell'Università e della Ricerca, by the project FAR 2010 "Geometria e Topologia" founded by University of Milano-Bicocca, and by Istituto Nazionale di Alta Matematica (GNSAGA).

Francesco Bastianelli

francesco.bastianelli@uniba.it

Edoardo Ballico

edoardo.ballico@unitn.it

Luca Benzo

luca.benzo@polito.it

1 Dipartimento di Matematica, Università degli Studi di Trento, Via Sommarive 14, 38123 Povo, TN, Italy

2 Dipartimento di Matematica, Università degli Studi di Bari, Via Edoardo Orabona 4, 70125 Bari, Italy

3 Dipartimento di Scienze Matematiche "Giuseppe Luigi Lagrange”, Politecnico di Torino, Corso Duca degli Abruzzi 24, 10129 Turin, Italy 


\section{Introduction}

The study of theta-characteristics on algebraic curves has been developed along various directions concerning, for instance, the dimension of spaces of global sections, or the geometry of effective divisors in the associated linear series. Consider a smooth complex projective curve $C$ of genus $g \geq 2$, and let $\mathcal{M}_{g}$ be the moduli space of smooth curves of genus $g$. We recall that a theta-characteristic $L$ is a line bundle on $C$ such that $L^{\otimes 2} \cong \omega_{C}$, and its parity is the residue modulo 2 of the dimension $h^{0}(C, L)$ of the space of global sections.

In the seminal paper [18], Mumford introduced a purely algebraic approach to thetacharacteristics, and he proved that the parity is invariant under flat deformations of pairs $(C, L)$. Along these lines, Harris [15] focused on the loci $\mathcal{M}_{g}^{r}$ in $\mathcal{M}_{g}$ of curves admitting a large theta-characteristic $L$, that is $h^{0}(C, L) \geq r+1$ and $h^{0}(C, L) \equiv r+1(\bmod 2)$ for some fixed integer $r \geq 0$. In particular, he showed that the codimension of each irreducible component of $\mathcal{M}_{g}^{r}$ is at most $\frac{r(r+1)}{2}$. The geometry of these loci has been thoroughly investigated (see, e.g. $[8,12,13,19,27])$, and recently, Harris' bound has been proved to be sharp for any value of $r \geq 0$ and $g \geq g(r)$, where $g(r)$ is an integer depending on $r$ (cf. [4,5]).

On the other hand, Kontsevich and Zorich [17] set their analysis of theta-characteristics in the moduli space of abelian differentials, which parameterizes isomorphism classes of pairs $(C, \omega)$ consisting of a smooth curve $C$ of genus $g$ endowed with a nonzero holomorphic form $\omega \in H^{0}\left(C, \omega_{C}\right)$. Given any partition $\underline{k}=\left(k_{1}, \ldots, k_{n}\right)$ of the integer $g-1$-i.e. a sequence of integers $k_{1} \geq \cdots \geq k_{n}>0$ such that $\sum_{i=1}^{n} k_{i}=g-1$-they obtain relevant results on the number of connected components of the locus $\mathcal{H}_{g}(2 \underline{k})$ described by pairs $(C, \omega)$ such that $\omega$ vanishes along a divisor of the form $2\left(k_{1} p_{1}+\cdots+k_{n} p_{n}\right)$, so that $\mathcal{O}_{C}\left(\sum_{i=1}^{n} k_{i} p_{i}\right)$ is a theta-characteristic on $C$ (see also $[14,21]$ for further results on these loci).

In this paper, we consider both the viewpoints above at once, and we focus on the subvarieties of the moduli space $\mathcal{M}_{g, n}$ described by $n$-pointed curves of genus $g$ admitting a large theta-characteristic with a global section vanishing with prescribed multiplicities at the marked points. More precisely, given an integer $r \geq 0$ and a partition $\underline{k}=\left(k_{1}, \ldots, k_{n}\right)$ of $g-1$, we are aimed at studying the loci defined as

$$
\mathcal{G}_{g}^{r}(\underline{k}):=\left\{\begin{array}{l|l}
{\left[C, p_{1}, \ldots, p_{n}\right] \in \mathcal{M}_{g, n}} & \begin{array}{l}
L:=\mathcal{O}_{C}\left(\sum_{i=1}^{n} k_{i} p_{i}\right) \text { is a } \\
\text { theta-characteristic with } h^{0}(C, L) \geq r+1 \\
\text { and } h^{0}(C, L) \equiv r+1(\bmod 2)
\end{array}
\end{array}\right\} .
$$

In particular, we achieve a general upper bound governing the codimension of $\mathcal{G}_{g}^{r}(\underline{k})$ in $\mathcal{M}_{g, n}$. Furthermore, for any $r \geq 0$ and $g$ greater than an explicit integer $g(r)$ depending on $r$, we present irreducible components of $\mathcal{G}_{g}^{r}(\underline{k})$ having maximal codimension, so that the bound turns out to be sharp.

Clearly, any locus $\mathcal{G}_{g}^{r}(\underline{k})$ maps on $\mathcal{M}_{g}^{r}$ under the forgetful morphism $\pi_{n}: \mathcal{M}_{g, n} \longrightarrow \mathcal{M}_{g}$. In particular, when $\underline{k}=(1, \ldots, 1), \mathcal{G}_{g}^{r}(\underline{k})$ dominates $\mathcal{M}_{g}^{r}$, and $\operatorname{dim} \mathcal{G}_{g}^{r}(\underline{k}) \geq \operatorname{dim} \mathcal{M}_{g}^{r}+r$ as the fibre over a general $[C] \in \mathcal{M}_{g}^{r}$ is described by the complete linear series $|L|$ associated to the large theta-characteristic on $C$. On the other hand, if $k=(g-1)$, then the subvarieties $\mathcal{G}_{g}^{r}:=\mathcal{G}_{g}^{r}(g-1)$ are the loci of subcanonical points, which recently gained renewed interest (see, e.g. [3,6,7]). Finally, it is worth noticing that the description of these subvarieties-or of corresponding loci in different moduli spaces-led to various applications in classical enumerative and projective geometry [15], in differential geometry [3,20], and in dynamical systems [17].

By means of Harris' bound, we prove the following. 
Theorem 1.1 Fix integers $r \geq 0, g>n>0$, and consider a partition $\underline{k}=\left(k_{1}, \ldots, k_{n}\right)$ of $g-1$. Then either $\mathcal{G}_{g}^{r}(\underline{k})$ is empty, or the codimension in $\mathcal{M}_{g, n}$ of each irreducible component $\mathcal{Z}$ of $\mathcal{G}_{g}^{r}(\underline{k})$ satisfies

$$
\operatorname{codim}_{\mathcal{M}_{g, n}} \mathcal{Z} \leq g-1+\frac{r(r-1)}{2}
$$

Accordingly, we shall say that an irreducible component $\mathcal{Z} \subset \mathcal{G}_{g}^{r}(\underline{k})$ has expected dimension if it satisfies equality in the latter bound, that is $\operatorname{dim} \mathcal{Z}=2 g-2+n-\frac{r(r-1)}{2}$. We note that when $\underline{k}=(1, \ldots, 1)$, Theorem 1.1 agrees with the bound on the codimension of $\mathcal{M}_{g}^{r}$, as it gives $\operatorname{dim} \mathcal{Z} \geq\left(3 g-3-\frac{r(r+1)}{2}\right)+r$. Moreover, the assertion for $\underline{k}=(g-1)$ coincides with [3, Theorem 1.1], and the proof of our result relies on a similar argument.

For any $r \geq 0$, we then consider the integer $g(r)$ defined by

$$
g(r):= \begin{cases}2 & \text { for } r=0 \\
3 r & \text { for } 1 \leq r \leq 3 \\
\left(\begin{array}{c}
r+2 \\
2
\end{array}\right) & \text { for } r \geq 4 .\end{cases}
$$

We prove the sharpness of the bound in Theorem 1.1 for any $r \geq 0$ and $g \geq g(r)$. Namely,

Theorem 1.2 For any genus $g \geq g(r)$, and for any partition $\underline{k}=\left(k_{1}, \ldots, k_{n}\right)$ of $g-1$, the locus $\mathcal{G}_{g}^{r}(\underline{k})$ is non-empty, and there exists an irreducible component $\mathcal{Z} \subset \mathcal{G}_{g}^{r}(\underline{k})$ having expected dimension. In particular, at a general point $\left[C, p_{1}, \ldots, p_{n}\right] \in \mathcal{Z}$, the large thetacharacteristic $\mathcal{O}_{C}\left(\sum_{i=1}^{n} k_{i} p_{i}\right)$ possesses exactly $r+1$ independent global sections and, apart from the cases $(r, g)=(0,2)$ and $(1,3)$, the curve $C$ is non-hyperelliptic.

In the light of the relation between $\mathcal{M}_{g}^{r}$ and $\mathcal{G}_{g}^{r}(\underline{k})$, the assertion for $\underline{k}=(1, \ldots, 1)$ is well known for small values of $r$ (cf. [27]), and it is included in [4, Theorem 1.2] for arbitrary $r$. In particular, the value of $g(r)$ can be lowered in this case (see [5,12]). Besides, the statement in the case $\underline{k}=(g-1)$ with $0 \leq r \leq 3$ is covered by different results in $[3,6,17]$, so Theorem 1.2 extends them to arbitrary large values of $r$.

We note further that under the assumption $\underline{k}=(g-1)$, the bound in Theorem 1.1 is meaningful as long as the expected dimension is non-negative, that is $g \geq\left\lfloor\frac{r^{2}-r+5}{4}\right\rfloor$, which is hypothetically the best value for $g(r)$ when $r$ is large enough.

In order to prove Theorem 1.2, we firstly show that the whole statement follows from the assertion for $\underline{k}=(g-1)$ (see Theorem 2.3). Roughly speaking, this depends on the fact that each irreducible component of the subcanonical locus $\mathcal{G}_{g}^{r}$ may be thought as a degeneration of any $\mathcal{G}_{g}^{r}(\underline{k})$ (cf. also [17, Corollary 2]). Therefore, the assertion for small values of $r$ follows from known results on the loci of subcanonical points.

As far as arbitrary large values of $r$ are concerned, we argue by induction on $g$ and $r$ separately, in analogy with $[4,12]$ where the sharpness of Harris' bound is proved. Thanks to [3, Theorem 4.1], if $g(r)$ is an integer such that $\mathcal{G}_{g(r)}^{r}$ admits an irreducible component $\mathcal{Z}_{g(r)}$ having expected dimension, then for any $g \geq g(r)$, the locus $\mathcal{G}_{g}^{r}$ has an irreducible component with the same property. By using basic facts on Eisenbud-Harris' limit linear series $[9,11]$, we slightly improve the latter result in order to keep track of global sections (see Theorem 2.7).

In the spirit of [4], we then set $g(r):=\left(\begin{array}{c}r+2 \\ 2\end{array}\right)$, and we prove that if $\mathcal{Z}_{g(r-1)} \subset \mathcal{G}_{g(r-1)}^{r-1}$ is an irreducible component satisfying equality in Theorem 1.1, then there exists an irreducible component $\mathcal{Z}_{g(r)} \subset \mathcal{G}_{g(r)}^{r}$ which still has expected dimension (cf. Theorem 3.10), so that Theorem 1.2 follows. The proof of such a result is mainly based on deformation theory and 
Ran's description of Hilbert schemes of points on nodal curves [22-26]. In particular, we consider nodal reducible curves in $\mathbb{P}^{r}$ consisting of an elliptic normal curve suitably attached to a degenerate curve $C$ such that $[C] \in \mathcal{M}_{g(r-1)}^{r-1}$. It follows from [4], that these singular curves can be deformed to smooth curves $X \subset \mathbb{P}^{r}$ such that $[X] \in \mathcal{M}_{g(r)}^{r}$, where $\mathcal{O}_{X}(1)$ is the corresponding large theta-characteristic. Therefore, a pair $[X, p]$ lies on $\mathcal{G}_{g(r)}^{r}$, i.e. $p \in X$ is a subcanonical point of $X$, if and only if the divisor $(g(r)-1) p$ is cut out on $X$ by some hyperplane. So we extend in these terms the notion of being a subcanonical point on the nodal curves. Finally, we show that the nodal curves can be smoothed into a component of expected dimension, preserving the property of having a subcanonical point.

The paper is organized as follows. In Sect. 2 we prove Theorem 1.1, we reduce the proof of Theorem 1.2 to the case $k=(g-1)$, and we recall some preliminary results about loci of subcanonical points. Then Sect. 3 is entirely devoted to perform the induction on $r$, and to conclude the proof of Theorem 1.2.

\section{Loci of large theta-characteristics with prescribed vanishing}

In this section, we firstly aim at proving Theorem 1.1. Then we shall reduce the proof of Theorem 1.2 to the case of loci of subcanonical points, and we shall recall some preliminary results on this topic.

\subsection{Notation}

We work throughout over the field $\mathbb{C}$ of complex numbers. By curve we mean a complete connected reduced algebraic curve over $\mathbb{C}$. Given a variety $X$, we say that a property holds for a general point $x \in X$ if it holds on a non-empty open subset of $\mathrm{X}$.

As is customary, we denote by $\mathcal{M}_{g}$ the moduli space of smooth curves of genus $g$. Let $\mathcal{M}_{g, n}$ be the moduli space consisting of isomorphism classes of ordered $(n+1)$ tuples $\left(C, p_{1}, \ldots, p_{n}\right)$, where $[C] \in \mathcal{M}_{g}$ and $p_{1}, \ldots, p_{n} \in C$ are distinct points, and let $\pi_{n}: \mathcal{M}_{g, n} \longrightarrow \mathcal{M}_{g}$ be the forgetful morphism sending $\left[C, p_{1}, \ldots, p_{n}\right]$ to $[C]$.

Moreover, let $\mathcal{S}_{g}$ denote the moduli space of spin curves, which parameterizes pairs $[C, L]$ such that $[C] \in \mathcal{M}_{g}$ and $L$ is a theta-characteristic on $C$. Hence there is a natural map $\varphi: \mathcal{S}_{g} \longrightarrow \mathcal{M}_{g}$ of degree $2^{2 g}$ sending $[C, L]$ to $[C]$. According to the notation on $\mathcal{M}_{g}$, we define

$$
\mathcal{S}_{g}^{r}:=\left\{[C, L] \in \mathcal{S}_{g} \mid h^{0}(C, L) \geq r+1 \text { and } h^{0}(C, L) \equiv r+1(\bmod 2)\right\} .
$$

In addition, we consider the moduli space $\mathcal{S}_{g, n}$ of $(n+2)$-tuples $\left[C, L, p_{1}, \ldots, p_{n}\right]$ with $\left[C, p_{1}, \ldots, p_{n}\right] \in \mathcal{M}_{g, n}$ and $[C, L] \in \mathcal{S}_{g}$, and we denote by $\mathcal{S}_{g, n}^{r}$ the sublocus of $n$-pointed spin curves such that $[C, L] \in \mathcal{S}_{g}^{r}$.

\subsection{Bound on the codimension of $\mathcal{G}_{g}^{r}(\underline{k})$ in $\mathcal{M}_{g, n}$}

We are now going to prove Theorem 1.1. As in the proof of [3, Theorem 1.1], the result is achieved by intersecting cycles on the relative symmetric product of families of curves with large theta-characteristics.

Proof of Theorem 1.1 Let us fix integers $r \geq 0, g>n>0$, and consider a partition $\underline{k}=\left(k_{1}, \ldots, k_{n}\right)$ of $g-1$. Assuming that $\mathcal{G}_{g}^{r}(\underline{k})$ is non-empty, we consider a point 
$\left[C, p_{1}, \ldots, p_{n}\right] \in \mathcal{G}_{g}^{r}(\underline{k})$. Hence we want to prove that any irreducible component $\mathcal{Z} \subset \mathcal{G}_{g}^{r}(\underline{k})$ passing through $\left[C, p_{1}, \ldots, p_{n}\right]$ has dimension $\operatorname{dim} \mathcal{Z} \geq 2 g-2+n-\frac{r(r-1)}{2}$.

By assumption, the line bundle $L:=\mathcal{O}_{C}\left(\sum_{i=1}^{n} k_{i} p_{i}\right)$ is a theta-characteristic having $h^{0}(C, L) \geq r+1$ and $h^{0}(C, L) \equiv r+1(\bmod 2)$, so that $\left[C, L, p_{1}, \ldots, p_{n}\right] \in \mathcal{S}_{g, n}^{r}$. Therefore, we can consider a versal deformation family $\left(\mathcal{C} \stackrel{\phi}{\longrightarrow} U, \mathcal{L} \longrightarrow \mathcal{C}, U \stackrel{\rho_{i}}{\longrightarrow} \mathcal{C}\right)$ of the $n$-pointed curve $\left(C, L, p_{1}, \ldots, p_{n}\right)$ in $\mathcal{S}_{g, n}$. So there is a commutative diagram of finite maps

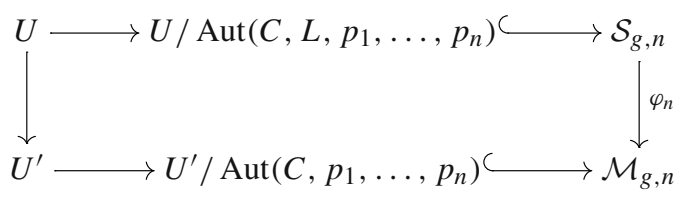

where $U^{\prime}$ is a versal deformation space of $\left(C, p_{1}, \ldots, p_{n}\right)$ in $\mathcal{M}_{g, n}$, and $\varphi_{n}$ is the natural map of degree $2^{2 g}$. In particular, the family $\phi: \mathcal{C} \longrightarrow U$ consists of smooth curves $C_{t}:=\phi^{-1}(t)$ of genus $g$, the line bundle $\mathcal{L} \longrightarrow \mathcal{C}$ restricts to a theta-characteristics $L_{t}:=\mathcal{L}_{\mid C_{t}}$ on each fibre, and for $i=1, \ldots, n$, the maps $\rho_{i}: U \longrightarrow \mathcal{C}$ are sections of $\phi$ with $p_{i, t}:=\rho_{i}(t) \in C_{t}$. Moreover, we may assume $\left(C_{0}, L_{0}, p_{1,0}, \ldots, p_{n, 0}\right)=\left(C, L, p_{1}, \ldots, p_{n}\right)$ for some point $0 \in U$.

Then we restrict the versal deformation to the locus $U^{r}:=\left\{t \in U \mid\left[C_{t}, L_{t}, p_{1, t}, \ldots, p_{n, t}\right] \in\right.$ $\left.\mathcal{S}_{g, n}^{r}\right\}$, and we consider the $(g-1)$-fold relative symmetric product $\mathcal{C}^{(g-1)} \stackrel{\Phi}{\longrightarrow} U^{r}$ of the family $\mathcal{C}$, so that the fibre over each $t$ is the $(g-1)$-fold symmetric product $C_{t}^{(g-1)}$ of the curve $C_{t}$. Let us define two subvarieties of $\mathcal{C}^{(g-1)}$ as

$$
\mathcal{P}:=\left\{k_{1} p_{1, t}+\cdots+k_{n} p_{n, t} \in C_{t}^{(g-1)} \mid t \in U^{r}\right\}
$$

which restricts to a point of the $\underline{k}$-diagonal on each fibre $C_{t}^{(g-1)}$, and

$$
\mathcal{Y}:=\left\{q_{1}+\cdots+q_{g-1} \in C_{t}^{(g-1)} \mid t \in U^{r} \text { and } \mathcal{O}_{C_{t}}\left(q_{1}+\cdots+q_{g-1}\right) \cong L_{t}\right\},
$$

which parameterizes effective divisors $Q_{t} \in C_{t}^{(g-1)}$ in the linear systems $\left|L_{t}\right|$. Thus, if $k_{1} p_{1, t}+\cdots+k_{n} p_{n, t} \in \mathcal{P} \cap \mathcal{Y}$, the line bundle $\mathcal{O}_{C_{t}}\left(\sum_{i=1}^{n} k_{i} p_{i, t}\right)$ is a theta-characteristic such that $h^{0}\left(C_{t}, \mathcal{O}_{C_{t}}\left(\sum_{i=1}^{n} k_{i} p_{i, t}\right)\right) \geq r+1$ and $h^{0}\left(C_{t}, \mathcal{O}_{C_{t}}\left(\sum_{i=1}^{n} k_{i} p_{i, t}\right)\right) \equiv r+$ $1(\bmod 2)$, that is $\left[C_{t}, p_{1, t}, \ldots, p_{n, t}\right] \in \mathcal{G}_{g}^{r}(\underline{k})$. It follows from the diagram (2.1), that the map $U \longrightarrow \mathcal{M}_{g, n}$ given by $t \longmapsto\left[C_{t}, p_{1, t} \ldots, p_{n, t}\right]$ is finite. Therefore, each irreducible component $\mathcal{Z} \subset \mathcal{G}_{g}^{r}(\underline{k})$ passing through $\left[C, p_{1}, \ldots, p_{n}\right]$ satisfies

$$
\operatorname{dim} \mathcal{Z} \geq \operatorname{dim} \mathcal{P}+\operatorname{dim} \mathcal{Y}-\operatorname{dim} \mathcal{C}^{(g-1)},
$$

which is the minimal dimension of any irreducible component of $\mathcal{P} \cap \mathcal{Y}$. We point out that $\operatorname{dim} \mathcal{P}=\operatorname{dim} U^{r} \geq 3 g-3+n-\frac{r(r+1)}{2}$ by Harris' bound (cf. [15, Corollary 1.11]). Moreover, $\operatorname{dim} \mathcal{Y} \geq \operatorname{dim} U^{r}+r$ and $\operatorname{dim} \mathcal{C}^{(g-1)}=\operatorname{dim} U^{r}+g-1$. Thus $\operatorname{dim} \mathcal{Z} \geq 2 g-2+n-$ $\frac{r(r-1)}{2}$, and the assertion follows.

We note that when the integer $g$ is small, the combinatorics of the loci $\mathcal{G}_{g}^{r}(\underline{k})$ is very simple. In particular, these loci can be easily described, and they always have expected dimension, as the following example shows (cf. [17, Theorem 2] and [6, Section 4]). 
Example 2.1 (Low genera) In the case $g=2$, the unique locus to consider is $\mathcal{G}_{2}^{0}(1)$. It is the irreducible divisor of $\mathcal{M}_{2,1}$ parameterizing pairs $[C, p]$, where $p \in C$ is a ramification point of the hyperelliptic map of $C$, and we have $h^{0}\left(C, \mathcal{O}_{C}(p)\right)=1$.

When $g=3$, each locus $\mathcal{G}_{3}^{r}(\underline{k})$ is still irreducible, with $0 \leq r \leq 1$ and $\underline{k} \in\{(1,1),(2)\}$. Recall that the canonical model of a non-hyperelliptic curve is a quartic curve in $C \subset \mathbb{P}^{2}$, and theta-characteristics are cut out by bitangent lines. Therefore, $\mathcal{G}_{3}^{0}(1,1)$ is the 6-dimensional locus described by triples $\left(C, p_{1}, p_{2}\right)$ such that $p_{1}$ and $p_{2}$ have the same tangent line, whereas $\mathcal{G}_{3}^{0}(2)$ has dimension 5 and parameterizes plane quartics endowed with a 4-inflection point. On the other hand, hyperelliptic curves of genus 3 give rise to $\mathcal{G}_{3}^{1}(1,1)$ and $\mathcal{G}_{3}^{1}(2)$. In particular, the former locus has dimension 6 and consists of triples $\left[C, p_{1}, p_{2}\right]$, where $p_{1}$ and $p_{2}$ are conjugated under the hyperelliptic involution, whereas $\mathcal{G}_{3}^{1}(2)$ is the 5-dimensional variety described by pairs $[C, p]$, where $p \in C$ is a Weierstrass point. Furthermore, it is easy to see that for any point $\left[C, p_{1}, p_{2}\right] \in \mathcal{G}_{3}^{r}(1,1)$ (resp. $\left.[C, p] \in \mathcal{G}_{3}^{r}(2)\right)$ as above, we have $h^{0}\left(C, \mathcal{O}_{C}\left(p_{1}+p_{2}\right)\right)=r+1\left(\operatorname{resp} . h^{0}\left(C, \mathcal{O}_{C}(p)\right)=r+1\right)$.

We note finally that if $p_{1}, p_{2} \in C$ are distinct Weierstrass points of a hyperelliptic curve $C$ of genus 3 , then $\left[C, p_{1}, p_{2}\right] \in \mathcal{G}_{3}^{0}(1,1)$, and these triples span a divisor of $\mathcal{G}_{3}^{0}(1,1)$.

We point out that the locus $\mathcal{M}_{g}^{r}$ is empty if and only if $r>\frac{g-1}{2}$ (see, e.g. [27]). This fact follows from Clifford's Theorem and from the description of linear series on hyperelliptic curves (cf. [1]). In particular, for any $0 \leq r \leq\left\lfloor\frac{g-1}{2}\right\rfloor$, hyperelliptic curves of genus $g$ possess theta-characteristics with exactly $r+1$ global sections. The example below shows that hyperelliptic curves provide several examples of large theta-characteristics with prescribed vanishing. However, the loci they describe may not have expected dimension, and they do not cover all the possibilities for $r$ and $\underline{k}$. This is indeed one of the main reasons for focusing on non-hyperelliptic curves.

Example 2.2 (Hyperelliptic locus) Let $L$ be a theta-characteristic on a hyperelliptic curve $C$ of genus $g$. Effective divisors in the linear series $|L|$ have the form $\sum \alpha_{i} w_{i}+\sum \beta_{j}\left(p_{j}+q_{j}\right)$, where each $w_{i}$ is a different Weierstass point, the distinct pairs $\left(p_{j}, q_{j}\right) \in C^{2}$ consist of points conjugated by the hyperelliptic involution, and the positive integers $\alpha_{i}, \beta_{j}$ are such that $\sum \alpha_{i}+2 \sum \beta_{j}=g-1$. In particular, the dimension of $|L|$ is $r=$ $\sum\left\lfloor\frac{\alpha_{i}}{2}\right\rfloor+\sum \beta_{j}$ (cf. [1, p. 13] and [17, Appendix B]). Then, if $\underline{k}$ is the partition of $g-1$ obtained by reordering $\left(\alpha_{1}, \ldots, \alpha_{s}, \beta_{1}, \beta_{1}, \ldots, \beta_{t}, \beta_{t}\right)$, the corresponding tuples of the form $\left(C, w_{1}, \ldots, w_{s}, p_{1}, q_{1}, \ldots, p_{t}, q_{t}\right)$ describe a sublocus of $\mathcal{G}_{g}^{r}(\underline{k})$ of dimension $2 g-1+t$. As it has been observed at the end of Example 2.1 - under the assumption $g=3, s=2$ and $t=0$ - such a sublocus may not be a whole component of $\mathcal{G}_{g}^{r}(\underline{k})$.

Furthermore, if for instance $\underline{k}=(g-1)$, hyperelliptic curves describe an irreducible component of $\mathcal{G}_{g}^{r}(g-1)$ consisting of pairs $\left[C, w_{1}\right]$, so that its dimension is $2 g-1$ and $r=\left\lfloor\frac{g-1}{2}\right\rfloor$. In particular, choosing $\rho \in\{0,1\}$ with the same parity as $r+1$, such a component may be viewed as an irreducible component of $\mathcal{G}_{g}^{\rho}(g-1)$ having expected dimension. However, no components with different parity can contain hyperelliptic curves.

\subsection{Reduction to the case of loci of subcanonical points}

In this subsection, we argue as in Theorem 1.1 and we show that, in order to prove Theorem 1.2, it is enough to focus on the partition $\underline{k}=(g-1)$.

We recall that the set of partitions $\underline{k}$ of $g-1$ is naturally endowed with the structure of partially ordered set, as follows. Given two partitions $\underline{k}=\left(k_{1}, \ldots, k_{n}\right)$ and $\underline{h}=\left(h_{1}, \ldots, h_{m}\right)$, 
we have that $\underline{k} \preceq \underline{h}$ if and only if there exists a partition $I_{1} \cup \cdots \cup I_{m}$ of the set of indices $I=\{1, \ldots, n\}$ such that $h_{j}:=\sum_{i \in I_{j}} k_{i}$ for any $j=1, \ldots, m$. For instance, we have that $\underline{k} \preceq(g-1)$ and $(1, \ldots, 1) \preceq \underline{k}$ for any partition $\underline{k}$ of $g-1$ : the former relation is obtained by taking the partition $I_{1}=I$, the latter one by partitioning the set $\{1, \ldots, g-1\}$ as $\left\{1, \ldots, k_{1}\right\} \cup\left\{k_{1}+1, \ldots, k_{1}+k_{2}\right\} \cup \cdots \cup\left\{k_{n-1}+1, \ldots, g-1\right\}$.

We prove the following.

Theorem 2.3 Let $\underline{h}=\left(h_{1}, \ldots, h_{m}\right)$ be a partition of $g-1$, and assume that there exists an irreducible component $\mathcal{Z} \subset \mathcal{G}_{g}^{r}(\underline{h})$ having expected dimension. Then for any partition $\underline{k}=\left(k_{1}, \ldots, k_{n}\right)$ satisfying $\underline{k} \preceq \underline{h}$, there exists an irreducible component $\mathcal{W} \subset \mathcal{G}_{g}^{r}(\underline{k})$ having expected dimension.

Furthermore, if the general point $\left[C, p_{1}, \ldots, p_{m}\right] \in \mathcal{Z}$ consists of a non-hyperelliptic curve $C$ such that $h^{0}\left(C, \mathcal{O}_{C}\left(\sum_{j=1}^{m} h_{j} p_{j}\right)\right)=r+1$, then the general point $\left[D, q_{1}, \ldots, q_{n}\right] \in \mathcal{W}$ parameterizes a non-hyperelliptic curve $D$ such that $h^{0}\left(D, \mathcal{O}_{D}\left(\sum_{i=1}^{n} k_{i} q_{i}\right)\right)=r+1$, as well.

In particular, if the subcanonical locus $\mathcal{G}_{g}^{r}(g-1)$ admits an irreducible component of expected dimension with general point as above, then $\mathcal{G}_{g}^{r}(\underline{k})$ does for any partition $\underline{k}$ of $g-1$.

Proof We notice that for any partition $\underline{k}$ satisfying $\underline{k} \preceq \underline{h}$, there exists a chain of relations $\underline{k} \preceq \underline{k}^{n-1} \preceq \cdots \preceq \underline{k}^{m+1} \preceq \underline{h}$ such that for any $n-1 \geq l \geq m+1$, the sequence $\underline{k}^{l}$ consists of exactly $l$ integers. Thus it suffices to prove the statement in the case $n=m+1$, so that the whole assertion follows by iteration. Accordingly, we assume hereafter that $n=m+1$. Consider a point $\left[C, p_{1}, \ldots, p_{m}\right] \in \mathcal{Z} \subset \mathcal{G}_{g}^{r}(\underline{h})$, and let $(C, L)$ be the associated spin curve, where $L:=\mathcal{O}_{C}\left(\sum_{j=1}^{m} h_{j} p_{j}\right)$. As in the proof of Theorem 1.1, we consider a versal deformation family $(\mathcal{C} \stackrel{\phi}{\longrightarrow} U, \mathcal{L} \longrightarrow \mathcal{C})$ of $(C, L)$ in the moduli space $\mathcal{S}_{g}$ of spin curves. Moreover, we set $U^{r}:=\left\{t \in U \mid\left[C_{t}, L_{t}\right] \in \mathcal{S}_{g}^{r}\right\}$ and $\mathcal{Y}:=\left\{q_{1}+\cdots+q_{g-1} \in C_{t}^{(g-1)} \mid t \in U^{r}\right.$ and $\left.\mathcal{O}_{C_{t}}\left(q_{1}+\cdots+q_{g-1}\right) \cong L_{t}\right\}$, and we denote by $\Delta_{\underline{k}}:=\left\{k_{1} q_{1}+\cdots+k_{n} q_{n} \in C_{t}^{(g-1)} \mid t \in U^{r}\right\}$ the relative $k$-diagonal. By the very same argument of Theorem 1.1, we deduce that the dimension of any irreducible component $\mathcal{W}_{\underline{k}}$ of $\mathcal{Y} \cap \Delta_{\underline{k}}$ is bounded by

$$
\operatorname{dim} \mathcal{W}_{\underline{k}} \geq \operatorname{dim} \mathcal{Y}+\operatorname{dim} \Delta_{\underline{k}}-\operatorname{dim} \mathcal{C}^{(g-1)} \geq 2 g-2+n-\frac{r(r-1)}{2} .
$$

We recall that $\underline{k} \preceq \underline{h}$ and $n=m+1$. Therefore, the relative $\underline{h}$-diagonal $\Delta_{h}$ is a codimension 1 subvariety of $\Delta_{\underline{k}}$, and the analogue of (2.3) gives $\operatorname{dim}\left(\mathcal{Y} \cap \Delta_{\underline{h}}\right) \geq 2 g-2+m-\frac{r(r-1)}{2}$, with $m=n-1$. We point out that, if $h_{1} q_{1}+\cdots+h_{m} q_{m} \in \mathcal{Y} \cap \Delta_{\underline{h}}$ and the $q_{j}$ 's are distinct, the isomorphism class $\left[C_{t}, q_{1}, \ldots, q_{m}\right] \in \mathcal{M}_{g, m}$ lies in $\mathcal{G}_{m}^{r}(\underline{h})$. Moreover, any component of $\mathcal{Y} \cap \Delta_{h}$ passing through $h_{1} q_{1}+\cdots+h_{m} q_{m}$ admits a finite rational map on an irreducible component of $\mathcal{G}_{m}^{r}(\underline{h})$. Since the component $\mathcal{Z} \in \mathcal{G}_{m}^{r}(\underline{h})$ has expected dimension $2 g-2+m-$ $\frac{r(r-1)}{2}$ and the point $\left[C, p_{1}, \ldots, p_{m}\right] \in \mathcal{Z}$ is such that $h_{1} p_{1}+\cdots+h_{m} p_{m} \in C^{(g-1)} \subset \mathcal{Y} \cap \Delta_{\underline{h}}$, there exists an irreducible component $\mathcal{Z}_{\underline{h}} \subset \mathcal{Y} \cap \Delta_{\underline{h}}$ admitting a finite dominant rational map $\mathcal{Z}_{\underline{h}} \rightarrow \mathcal{Z}$ given by $h_{1} q_{1}+\cdots+h_{m} q_{m} \in C_{t}^{(g-1)} \longmapsto\left[C_{t}, q_{1}, \ldots, q_{m}\right]$.

Let $\mathcal{W}_{\underline{k}}$ be an irreducible component of $\mathcal{Y} \cap \Delta_{\underline{k}}$ containing $\mathcal{Z}_{\underline{h}}$. By inequality (2.3), we have $\operatorname{dim} \overline{\mathcal{W}}_{\underline{k}}>\operatorname{dim} \mathcal{Z}_{\underline{h}}=2 g-3+n-\frac{r(r-1)}{2}$. Hence the general point $k_{1} q_{1}+\cdots+k_{n} q_{n} \in \mathcal{W}_{\underline{k}}$ is such that the $q_{i}$ 's are distinct. Thus there exists a finite dominant rational map $\mathcal{W}_{\underline{k}}-\rightarrow$ $\mathcal{W} \subset \mathcal{M}_{g, n}$ defined as $k_{1} q_{1}+\cdots+k_{n} q_{n} \in C_{t}^{(g-1)} \longmapsto\left[C_{t}, q_{1}, \ldots, q_{n}\right]$, whose image is dense into an irreducible component $\mathcal{W}$ of $\mathcal{G}_{g}^{r}(\underline{k})$. Then, we need to prove that $\mathcal{W}_{\underline{k}}$-and 
hence $\mathcal{W}$-has the expected dimension, that is $\operatorname{dim} \mathcal{W}_{\underline{k}}=\operatorname{dim} \mathcal{Z}_{h}+1$. As $\mathcal{W}_{k} \subset \mathcal{Y} \cap \Delta_{\underline{k}}$ is an irreducible component, $\Delta_{\underline{h}}$ has codimension 1 in $\Delta_{\underline{k}}^{-}$, and $\mathcal{Z}_{\underline{h}}$ is an irreducible component of $\mathcal{W}_{\underline{k}} \cap \Delta_{\underline{h}}$, the first part of the assertion follows.

In order to conclude the proof, we assume that $\left[C, p_{1}, \ldots, p_{m}\right] \in \mathcal{Z}$ consists of a nonhyperelliptic curve $C$ such that $h^{0}\left(C, \mathcal{O}_{C}\left(\sum_{j=1}^{m} h_{j} p_{j}\right)\right)=r+1$. Let $U^{\prime} \subset U^{r}$ be the image of $\mathcal{W}_{\underline{k}}$ under the morphism $\mathcal{C}^{(g-1)} \stackrel{\Phi}{\longrightarrow} U^{r}$, that is $U^{\prime}:=\left\{t \in U^{r} \mid \exists q_{1}, \ldots, q_{n} \in\right.$ $C_{t}$ with $\left.k_{1} q_{1}+\cdots+k_{n} q_{n} \in \mathcal{W}_{\underline{k}}\right\}$, and let $\left(\mathcal{C} \stackrel{\phi^{\prime}}{\longrightarrow} U^{\prime}, \mathcal{L} \longrightarrow \mathcal{C}\right)$ be the restriction of the versal deformation family of $\left(C, \mathcal{O}_{C}\left(\sum_{j=1}^{m} h_{j} p_{j}\right)\right)$. Therefore, for the general point $t \in U^{\prime}$, there exist $q_{1}, \ldots, q_{n} \in C_{t}$ such that $\left[C_{t}, q_{1}, \ldots, q_{n}\right] \in \mathcal{W}$ and $L_{t} \cong \mathcal{O}_{C_{t}}\left(\sum_{i=1}^{n} k_{i} q_{i}\right)$. Moreover, $h_{1} p_{1}+\cdots+h_{m} p_{m} \in \mathcal{Z}_{\underline{h}} \subset \mathcal{W}_{\underline{k}}$, and hence $0 \in U^{\prime}$, i.e. the curve $C=C_{0}$ is a special fibre of the family $\mathcal{C} \stackrel{\phi^{\prime}}{\longrightarrow} U^{\prime}$ with theta-characteristic $L_{0} \cong \mathcal{O}_{C}\left(\sum_{j=1}^{m} h_{j} p_{j}\right)$. Thus the general fibre $C_{t}=\left(\phi^{\prime}\right)^{-1}(t)$ cannot be hyperelliptic if $C$ is not. Furthermore, upper semi-continuity of the function $t \longmapsto h^{0}\left(C_{t}, L_{t}\right)$ implies that $h^{0}\left(C_{t}, \mathcal{O}_{C_{t}}\left(\sum_{i=1}^{n} k_{i} q_{i}\right)\right)=r+1$ for general $t \in U^{\prime}$ (see, e.g. [16, Theorem III.12.8]).

Remark 2.4 We would like to note that the very same argument of Theorem 2.3 provides an alternative criterion for proving that the $\mathcal{G}_{g}^{r}(\underline{k})$ 's admit components with expected dimension. Namely, assume that $\mathcal{Z} \subset \mathcal{M}_{g}^{r}$ is an irreducible component having expected dimension, and for some $2 \leq m \leq g-1$, let $\underline{k}^{g-1}=(1, \ldots, 1) \preceq \underline{k}^{g-2} \preceq \cdots \preceq \underline{k}^{m}$ be a chain of partitions of $g-1$ such that for all $g-1 \geq j \geq m$, the sequence $\underline{k}^{j}$ consists of $j$ integers. If for any such a $j$, there exists $\left[C, p_{1}, \ldots, p_{j}\right] \in \mathcal{G}_{g}^{r}\left(\underline{k}^{j}\right)$ with $\pi_{j}\left(\left[C, p_{1}, \ldots, p_{j}\right]\right) \in \mathcal{Z}$, then each $\mathcal{G}_{g}^{r}\left(\underline{k}^{j}\right)$ admits a component having expected dimension. In particular, if $m=g-1$, then the locus $\mathcal{G}_{g}^{r}(g-1)$ does.

\subsection{Loci of subcanonical points}

Consider a smooth projective curve $C$ of genus $g \geq 2$. We recall that a point $p \in C$ is a subcanonical point if the line bundle $\mathcal{O}_{C}((2 g-2) p)$ is isomorphic to the canonical bundle $\omega_{C}$, that is $[C, p] \in \mathcal{G}_{g}^{r}(g-1)$ for some $r \geq 0$. We denote by $\mathcal{G}_{g} \subset \mathcal{M}_{g, 1}$ the locus parameterizing pairs $[C, p]$ such that $p \in C$ is a subcanonical point, and we set hereafter $\mathcal{G}_{g}^{r}:=\mathcal{G}_{g}^{r}(g-1)$.

When $2 \leq g \leq 3$, the loci of subcanonical points have been already described in Example 2.1. If instead $g \geq 4$, Kontsevich and Zorich [17] proved that the locus $\mathcal{G}_{g}$ consists of three irreducible components having dimension $2 g-1$. The component $\mathcal{G}_{g}^{\text {hyp }}$ parameterizes hyperelliptic curves endowed with a Weierstrass point (cf. Example 2.2), whereas the remaining components, $\mathcal{G}_{g}^{\text {odd }}$ and $\mathcal{G}_{g}^{\text {even }}$, are described by non-hyperelliptic curves such that $h^{0}\left(C, \mathcal{O}_{C}((g-1) p)\right)$ is odd and even, respectively. Moreover, Bullock [6] showed that the general point $[C, p] \in \mathcal{G}_{g}^{\text {odd }}\left(\operatorname{resp} .[C, p] \in \mathcal{G}_{g}^{\text {even }}\right)$ is such that $h^{0}\left(C, \mathcal{O}_{C}((g-1) p)\right)=1$ (resp. $\left.h^{0}\left(C, \mathcal{O}_{C}((g-1) p)\right)=2\right)$.

By collecting these facts, [3, Theorems 1.2 and 1.3] and [3, Corollary 4.5], we obtain the following.

Proposition 2.5 Let $0 \leq r \leq 3$ and let $g(r)$ be defined by (1.1). For any genus $g \geq g(r)$, there exists an irreducible component $\mathcal{Z} \subset \mathcal{G}_{g}^{r}$ having expected dimension. Moreover, the 
general point $[C, p] \in \mathcal{Z}$ satisfies $h^{0}\left(C, \mathcal{O}_{C}((g-1) p)\right)=r+1$ and, apart from the cases $(r, g)=(0,2)$ and $(1,3)$, the curve $C$ is non-hyperelliptic.

The following example describes the case $r=2$ with $g=g(r)=6$, and it provides the base case of the induction on $r$ we shall perform in the next section.

Example 2.6 ( $r=2$ and $g=6$ ) It is well known that the locus $\mathcal{M}_{6}^{2}$ is irreducible of pure dimension 12, and its general point parameterizes a smooth curve $C \subset \mathbb{P}^{2}$ of degree 5 (see, e.g. [19, p. 293]). Moreover, if $C \subset \mathbb{P}^{2}$ is such a curve, we have that $\omega_{C} \cong \mathcal{O}_{C}(2)$, and $L:=\mathcal{O}_{C}(1)$ is the unique theta-characteristic on $C$ with $h^{0}(C, L)=3$. Therefore, given a plane quintic curve $C \subset \mathbb{P}^{2}$ and a partition $\underline{k}=\left(k_{1}, \ldots, k_{n}\right)$ of $g-1=5$, we have $\left[C, p_{1}, \ldots, p_{n}\right] \in \mathcal{G}_{6}^{2}(\underline{k})$ if and only if the divisor $k_{1} p_{1}+\cdots+k_{n} p_{n}$ is cut out on $C$ by some line $\ell \subset \mathbb{P}^{2}$.

As far as the case $\underline{k}=(5)$ is concerned, we have that the locus of subcanonical points $\mathcal{G}_{6}^{2} \subset \mathcal{M}_{6,1}$ admits an irreducible component $\mathcal{Z}_{6}$ with expected dimension $2 g-2=10$, whose general point $[C, p]$ parameterizes a smooth (non-hyperelliptic) quintic curve $C \subset \mathbb{P}^{2}$ having a 5-fold inflection point at $p \in C$, and $h^{0}\left(C, O_{C}((g-1) p)\right)=3$. For the sake of completeness, we include a proof of this fact (cf. also [6, Section 4.5]).

Considering a point and a line $p \in \ell \subset \mathbb{P}^{2}$, it is elementary to check that the condition mult $(C, \ell)_{p} \geq 5$ imposes exactly 5 independent linear conditions to the linear system $\left|\mathcal{O}_{\mathbb{P}^{2}}(5)\right|$ parameterizing plane quintic curves. We note that the linear subsystem $V(p, \ell) \subset\left|\mathcal{O}_{\mathbb{P}^{2}}(5)\right|$ of curves satisfying the condition mult $(C, \ell)_{p} \geq 5$ is non-empty as, for instance, it contains reducible curves $C_{0}=\ell \cup \Gamma$ consisting of $\ell$ and a general quartic $\Gamma \subset \mathbb{P}^{2}$. Of course $p$ is the only base point of $V(p, \ell)$ (consider, e.g. the union of five general lines through $p$ ), and being $C_{0}$ smooth at $p$, Bertini's Theorem ensures that the general element of $V(p, \ell)$ is smooth. So we consider the irreducible variety $\Lambda:=\left\{(p,[\ell]) \in \mathbb{P}^{2} \times\left(\mathbb{P}^{2}\right)^{*} \mid p \in \ell\right\}$ and we define

$$
\mathcal{V}:=\left\{(C,(p,[\ell])) \in\left|\mathcal{O}_{\mathbb{P}^{2}}(5)\right| \times \Lambda \mid \operatorname{mult}(C, \ell)_{p} \geq 5\right\}
$$

Then the second projection $\mathcal{V} \stackrel{\pi_{2}}{\longrightarrow} \Lambda$ endows $\mathcal{V}$ of the structure of $\left(\mathbb{P}^{15}\right)$-bundle since for any $(p,[\ell]) \in \Lambda$, we have that $\pi_{2}^{-1}(p,[\ell])=V(p, \ell) \cong \mathbb{P}^{15}$. In particular, $\mathcal{V}$ is an irreducible variety of dimension $\operatorname{dim} \mathcal{V}=\operatorname{dim} \Lambda+15=18$. Finally, we consider the modular map $\mathcal{V} \rightarrow \mathcal{M}_{6,1}^{2}$ sending a triple $(C,(p,[\ell]))$, with $C$ smooth, to the corresponding point $[C, p] \in \mathcal{M}_{6,1}$. Since $C$ possesses finitely many subcanonical points and the isomorphism class of a smooth plane curve is described by the action of PGL(3), we conclude that the image of $\mathcal{V}$ in $\mathcal{M}_{6,1}^{2}$ is dense into an irreducible component $\mathcal{Z}_{6} \subset \mathcal{G}_{6}^{2}$ of dimension $\operatorname{dim} \mathcal{V}-$ $\operatorname{dim} \operatorname{PGL}(3)=10$.

Before stating the last result of this section, we need to recall few basic facts concerning Eisenbud-Harris' theory of limit linear series (see [9,11]). Let $\mathfrak{l}=(L, V)$ be a $\mathfrak{g}_{d}^{r}$ on the curve $C$, that is a line bundle $L$ of degree $d$ endowed with a $(r+1)$ dimensional subspace $V \subset H^{0}(C, L)$. Given a point $p \in C$, the set $\left\{\operatorname{ord}_{p}(s) \mid s \in V\right\}$ of orders of vanishing of sections of $V$ at $p$ consists of exactly $r+1$ distinct integers $0 \leq a_{0}^{\mathfrak{l}}(p)<a_{1}^{\mathfrak{l}}(p)<\cdots<a_{r}^{\mathfrak{l}}(p) \leq d$, and the sequence $\underline{a}^{\mathfrak{l}}(p):=\left(a_{0}^{\mathfrak{l}}(p), a_{1}^{\mathfrak{l}}(p), \ldots\right.$, $\left.a_{r}^{\mathfrak{l}}(p)\right)$ is called the vanishing sequence of $\mathfrak{l}$ at $p$. When $\mathfrak{l}$ is the complete canonical series $K_{C}:=\left(\omega_{C}, H^{0}\left(C, \omega_{C}\right)\right)$, the sequence $\left(t_{0}(p), \ldots, t_{g-1}(p)\right)$ such that $t_{i}(p):=a_{i}^{K_{C}}(p)+1$ equals the sequence of Weierstrass gaps of $p$, which is the increasing sequence of integers $\left\{0 \leq n \leq 2 g-1 \mid h^{0}\left(C, \mathcal{O}_{C}((n-1) p)\right)=h^{0}\left(C, \mathcal{O}_{C}(n p)\right)\right\}$. In particular, a point $p \in C$ 
is subcanonical if and only if $a_{g-1}^{\mathfrak{l}}(p)=2 g-2$. Furthermore, $h^{0}\left(C, \mathcal{O}_{C}((g-1) p)\right)=r+1$ for some $r \geq 0$, if and only if the set $\left\{i \mid a_{i}^{\mathfrak{l}}(p) \leq g-2\right\}$ consists of exactly $g-1-r$ elements.

So we consider a reducible curve $X=C \cup_{q} E$ consisting of two smooth curves $C$ and $E$ of genus $g-1$ and 1 respectively, meeting at a single ordinary node $q$. A (refined) limit $\mathfrak{g}_{d}^{r}$ on $X$ is a collection $\mathfrak{l}=\left\{\left(L_{C}, V_{C}\right),\left(L_{E}, V_{E}\right)\right\}$, where $\mathfrak{l}_{C}:=\left(L_{C}, V_{C}\right)$ and $\mathfrak{l}_{E}:=\left(L_{E}, V_{E}\right)$ are $\mathfrak{g}_{d}^{r}$ on $C$ and $E$ respectively, such that the vanishing sequences $\underline{a}^{\mathfrak{l}_{C}}(q)$ and $\underline{a}^{\mathfrak{l}_{E}}(q)$ satisfy the compatibility conditions $a_{i}^{\mathfrak{l}_{C}}(q)+a_{r-i}^{\mathfrak{l}_{E}}(q)=d$ for any $0 \leq i \leq r$.

The following theorem follows from [3, Theorem 4.1 and Corollary 4.4], and it shall be involved in the proof of Theorem 1.2.

Theorem 2.7 Let $r \geq 1$ and assume that there exists an integer $g(r)$ such that $\mathcal{G}_{g(r)}^{r}$ admits an irreducible component $\mathcal{Z}_{g(r)}$ having expected dimension. Then for any $g \geq g(r)$, there exists an irreducible component $\mathcal{Z}_{g}$ of $\mathcal{G}_{g}^{r}$ having expected dimension, as well.

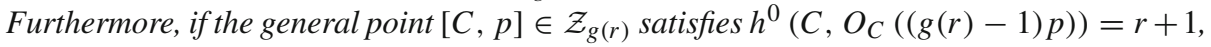
then also the general point of $\mathcal{Z}_{g}$ does.

Proof The first assertion of the theorem follows straightforwardly from [3, Theorem 4.1]. On the other hand, we want to prove that the general point $[C, p] \in \mathcal{Z}_{g}$ satisfies $h^{0}\left(C, O_{C}((g-1) p)\right)=r+1$. When $g=g(r)$ the statement is true by assumption. Hence we argue by induction on $g$, and we assume that the assertion holds true up to $g-1$. As in the proof of [3, Corollary 4.4], there exists a family $(\mathcal{X} \stackrel{\phi}{\longrightarrow} T, T \stackrel{\rho}{\longrightarrow} \mathcal{X})$ of smooth pointed curves such that $\left[X_{t}, p_{t}\right] \in \mathcal{Z}_{g}$ for any $t \neq 0$, and the central fibre $\left(X_{0}, p_{0}\right)=$ $\left(C \cup_{q} E, p\right)$ is given by a curve $[C, q] \in \mathcal{Z}_{g-1}$ attached at an ordinary node $q$ to an elliptic curve $E$, where $(p-q) \in E$ is a $(2 g-2)$-torsion point. Furthermore, the canonical bundles $\omega_{X_{t}} \cong \mathcal{O}_{X_{t}}\left((2 g-2) p_{t}\right)$ induce a limit $\mathfrak{g}_{2 g-2}^{g-1}$ on $C \cup_{q} E, \mathfrak{l}:=\left\{\left(L_{C}, V_{C}\right),\left(L_{E}, V_{E}\right)\right\}$, where $L_{C} \cong \omega_{C}(2 q) \cong \mathcal{O}_{C}((2 g-2) q)$ and $L_{E} \cong \mathcal{O}_{E}((2 g-2) q)$. In particular, since $\mathfrak{l}_{C}:=\left(L_{C}, V_{C}\right)$ is a base-point-free complete linear series, if $\underline{a}^{K_{C}}(q):=\left(a_{0}, \ldots, a_{g-2}\right)$ is the vanishing sequence of the canonical linear series $K_{C}$ at the subcanonical point $q \in C$, then $\underline{a}^{l_{C}}(q)=\left(0, a_{0}+2, \ldots, a_{g-2}+2\right)$, where $a_{g-2}+2=2 g-2$. We want to prove that $h^{0}\left(X_{t}, \mathcal{O}_{X_{t}}\left((g-1) p_{t}\right)\right)=r+1$ for general $t \in T$.

Compatibility conditions on limit linear series give $\underline{a}^{\mathfrak{l}_{E}}(q)=\left(2 g-4-a_{g-2}, \ldots, 2 g-4-\right.$ $\left.a_{g-2}, 2 g-2\right)$. Moreover, vanishing sequences of $\mathfrak{l}_{E}$ satisfy $a_{i}^{\mathfrak{l}_{E}}(p)+a_{g-1-i}^{\mathfrak{l}_{E}}(q) \leq 2 g-2$ for any $0 \leq i \leq g-1$. Therefore, $\underline{a}^{\mathfrak{l}_{E}}(p) \leq\left(0, a_{0}+2, \ldots, a_{g-2}+2\right)$ and, by upper semicontinuity of vanishing sequences (see, e.g. [10] and [3, Remark 2.2]), we deduce $\underline{a}^{K_{X_{t}}}\left(p_{t}\right) \leq$ $\underline{a}^{\mathfrak{l}_{E}}(p) \leq\left(0, a_{0}+2, \ldots, a_{g-2}+2\right)$ for general $t \in T$. We recall that $\left[X_{t}, p_{t}\right] \in \mathcal{G}_{g}^{r}$ for $t \neq 0$, hence $p_{t} \in X_{t}$ is a subcanonical point such that $h^{0}\left(X_{t}, \mathcal{O}_{X_{t}}\left((g-1) p_{t}\right)\right) \geq r+1$ and $h^{0}\left(X_{t}, \mathcal{O}_{X_{t}}\left((g-1) p_{t}\right)\right) \equiv r+1(\bmod 2)$. Besides, $h^{0}\left(C, O_{C}((g-2) p)\right)=r+1$ by induction, hence the set $\left\{i \mid a_{i} \leq g-3\right\}$ consists of exactly $g-2-r$ elements. Let $\underline{a}^{K_{X_{t}}}\left(p_{t}\right):=\left(c_{0}, \ldots, c_{g-1}\right)$ for general $t \in T$, and let $|\cdot|$ denote the cardinality of a set. Then

$$
\begin{aligned}
\left|\left\{j \mid c_{j} \leq g-2\right\}\right| & \geq 1+\left|\left\{i \mid a_{i}+2 \leq g-2\right\}\right| \\
& \geq 1+\left|\left\{i \mid a_{i} \leq g-3\right\}\right|-1=g-1-(r+1) .
\end{aligned}
$$

Thus $r+1 \leq h^{0}\left(X_{t}, \mathcal{O}_{X_{t}}\left((g-1) p_{t}\right)\right) \leq r+2$, and the assertion follows as $h^{0}\left(X_{t}, \mathcal{O}_{X_{t}}\left((g-1) p_{t}\right)\right) \equiv r+1(\bmod 2)$. 


\section{Existence of components with expected dimension}

In this section we shall prove Theorem 1.2. In the light of Theorem 2.3, Proposition 2.5, and Theorem 2.7, we essentially need to show that for any $r \geq 4$ and $g(r):=\left(\begin{array}{c}r+2 \\ 2\end{array}\right)$, there exists an irreducible component $\mathcal{Z}_{g(r)}$ with expected dimension of the locus $\mathcal{G}_{g(r)}^{r}$ of subcanonical points, and its general point $[C, p] \in \mathcal{Z}_{g(r)}$ satisfies $h^{0}\left(C, \mathcal{O}_{C}((g(r)-1) p)\right)=r+1$. Large part of the section shall indeed be devoted to prove this fact, which is included in Theorem 3.10. Actually, the proof shall be mainly set in the Hilbert scheme Hilb ${ }_{g(r), g(r)-1}^{r}$ of curves of arithmetic genus $g(r)$ and degree $g(r)-1$ in $\mathbb{P}^{r}$, with at most nodes as singularities.

Section 3.1 gives the main results of [4], assuring the existence of an irreducible component $W_{g(r)}^{r} \subset \operatorname{Hilb}_{g(r), g(r)-1}^{r}$ whose general point is a smooth curve $C \subset \mathbb{P}^{r}$ such that $\mathcal{O}_{C}(1)$ is a large theta-characteristic. Moreover, we shall slightly improve the description of curves parameterized over $W_{g(r)}^{r}$. In Sect. 3.2 we shall extend the notion of 'subcanonical point' to singular curves in $W_{g(r)}^{r}$, and we shall focus on certain reducible curves admitting such a 'limit subcanonical point'. In Section 3.3 we shall prove that those reducible curves can be deformed to smooth curves endowed with a subcanonical point, and we shall achieve the existence of components $\mathcal{Z}_{g(r)}$ as above. Finally, Section 3.4 shall be addressed to conclude the proof of Theorem 1.2.

\subsection{Components of $\mathcal{M}_{g}^{r}$ having expected dimension}

We summarize and slightly improve some of the results included in [4], where the sharpness of Harris' bound is proved.

For a fixed integer $r \geq 2$, we set hereafter $g=g(r):=\left(\begin{array}{c}r+2 \\ 2\end{array}\right)$, and we consider the locus $\mathcal{S}_{g}^{r}$ of pairs $[C, L]$ such that $L$ is a large theta-characteristic on $C$. Thanks to [4, Theorem 3.3], there exists an irreducible component $\mathcal{V}_{g}^{r} \subset \mathcal{S}_{g}^{r}$ having dimension $3 g-3-\frac{r(r+1)}{2}$, whose image under the finite morphism $\varphi: \mathcal{S}_{g} \longrightarrow \mathcal{M}_{g}$ is an irreducible component of $\mathcal{M}_{g}^{r}$ attaining equality in Harris' bound. In addition, for a general point $[C, L] \in \mathcal{V}_{g}^{r}$, the line bundle $L$ is very ample with $h^{0}(C, L)=r+1$, and the image of the embedding $C \stackrel{\varphi_{|L|}}{\longrightarrow} \mathbb{P}^{r}$ is a smooth curve-which we still denote by $C \subset \mathbb{P}^{r}$ - of degree $g-1$ such that the normal bundle $N_{C / \mathbb{P}^{r}}$ of $C$ in $\mathbb{P}^{r}$ satisfies $h^{1}\left(C, N_{C / \mathbb{P}^{r}}\right)=0$.

So we consider the Hilbert scheme Hilb ${ }_{g, g-1}^{r}$ parameterizing reduced curves of arithmetic genus $g$ and degree $g-1$ in $\mathbb{P}^{r}$ with at most nodes as singularities. Given a general point $[C, L] \in \mathcal{V}_{g}^{r}$ and the corresponding embedded curve $C \subset \mathbb{P}^{r}$, we denote by $W_{g}^{r} \subset$ Hilb $_{g, g-1}^{r}$ the (unique) irreducible component containing the point [C], and by $W_{g \text {,sm }}^{r} \subset W_{g}^{r}$ the dense open subset parameterizing smooth curves. The following result is a simple consequence of [5, Proposition 2.7].

Proposition 3.1 The component $W_{g}^{r} \subset$ Hilb $_{g, g-1}^{r}$ has dimension $\operatorname{dim} W_{g}^{r}=3 g-4+\left(\begin{array}{c}r+2 \\ 2\end{array}\right)$, and $\left[\Gamma, \mathcal{O}_{\Gamma}(1)\right] \in \mathcal{S}_{g}^{r}$ for any smooth curve $[\Gamma] \in W_{g(r), s m}^{r}$.

Proof By [5, Proposition 2.7], we have that $\operatorname{dim} W_{g}^{r}=3 g-4+\left(\begin{array}{c}r+2 \\ 2\end{array}\right)$, and for general [C] $\in$ $W_{g, \text { sm }}^{r}$, the line bundle $\mathcal{O}_{C}(1)$ is a very ample theta-characteristic such that $\left[C, \mathcal{O}_{C}(1)\right] \in$ $\mathcal{S}_{g(r)}^{r}$. Then, we consider the universal family $\mathcal{C} \subset \mathbb{P}^{r} \times W_{g, \mathrm{sm}}^{r} \stackrel{\psi}{\rightarrow} W_{g, \mathrm{sm}}^{r}$, together with the relative hyperplane bundle $\mathcal{O}_{\mathcal{C}}(1)$ and the relative quadric bundle $\mathcal{O}_{\mathcal{C}}(2)$, whose restrictions to the general fibre $C$ of $\psi$ are $\mathcal{O}_{C}(1)$ and $\mathcal{O}_{C}(2)$, respectively. Since $\mathcal{O}_{C}(1)$ is a thetacharacteristic, the canonical bundle on $C$ is $\omega_{C} \cong \mathcal{O}_{C}(2)$, and hence $h^{1}\left(C, \mathcal{O}_{C}(2)\right)=1$. 
By upper semi-continuity of cohomology of $\mathcal{O}_{\mathcal{C}}(2)$, we deduce that $h^{1}\left(\Gamma, \mathcal{O}_{\Gamma}(2)\right) \geq 1$ for any $[\Gamma] \in W_{g \text {,sm. }}^{r}$. We note that $\operatorname{deg} \mathcal{O}_{\Gamma}(2)=2 g-2$, as $\operatorname{deg} \Gamma=g-1$. Thus RiemannRoch Theorem assures that $h^{1}\left(\Gamma, \mathcal{O}_{\Gamma}(2)\right)=1$, and $\mathcal{O}_{\Gamma}(2) \cong \omega_{\Gamma}$. In particular, $\mathcal{O}_{\Gamma}(1)$ is a theta-characteristic, and the assertion follows.

Remark 3.2 In the light of Proposition 3.1, there is a natural modular map $v: W_{g \text {,sm }}^{r} \longrightarrow \mathcal{V}_{g}^{r}$ sending $[\Gamma] \in W_{g \text {,sm }}^{r}$ to $\left[\Gamma, \mathcal{O}_{\Gamma}(1)\right] \in \mathcal{S}_{g}^{r}$. We point out that, since any smooth curve possesses finitely many theta-characteristics, the dimension of the fibres of $v$ equals the dimension of the space PGL $(r+1)$ of projective transformations of $\mathbb{P}^{r}$, according to the fact that $\operatorname{dim} W_{g, \mathrm{sm}}^{r}=\operatorname{dim} \mathcal{V}_{g}^{r}+(r+1)^{2}-1$.

Turning to singular curves contained in $W_{g}^{r}=W_{g(r)}^{r}$, it follows from [4, Section 3] that for any $r \geq 3$, the component $W_{g}^{r}$ contains the locus $W_{g, h}^{r}$ described by all the reducible nodal curves $X=C \cup E \subset \mathbb{P}^{r}$ such that

(i) $C$ lies into a hyperplane $H \subset \mathbb{P}^{r}$ and, under the isomorphism $H \cong \mathbb{P}^{r-1},[C] \in$ $W_{g(r-1), \mathrm{sm}}^{r-1}$

(ii) $E$ is an elliptic normal curve of degree $g(r)-g(r-1)=r+1$;

(iii) $C$ and $E$ meet transversally at the 0 -dimensional scheme of length $r+1$ cut out by $H$ on $E$.

Furthermore, the locus $W_{g, h}^{r}$ is a divisorial component of $W_{g}^{r}$. Namely,

Proposition 3.3 For any $r \geq 3$ and $g=\left(\begin{array}{c}r+2 \\ 2\end{array}\right)$, the locus $W_{g, h}^{r}$ is equidimensional, and it has codimension 1 in $W_{g}^{r}$.

Proof We want to compute the number $n$ of parameters a general curve $X:=C \cup E$ parameterized by any irreducible component of $W_{g, h}^{r}$ depends on. Let $S:=C \cap E$ be the set of singularities of $X$, let $\mathcal{E} \subset$ Hilb $_{1, r+1}^{r}$ be the subscheme parameterizing smooth elliptic normal curves containing $S$, and let $R \subset \mathcal{E}$ be the irreducible component parameterizing [E] $\in \operatorname{Hilb}_{1, r+1}^{r}$. By [4, Lemma 3.1], the normal bundle $N_{E / \mathbb{P}^{r}}$ of $E$ in $\mathbb{P}^{r}$ satisfies $h^{1}\left(E, N_{E / \mathbb{P}^{r}}(-S)\right)=0$. Thus [2, Lemma 2.4] assures that $R$ is smooth at [E]. Since, by the same lemma, the tangent space to $R$ at $[E]$ is isomorphic to $H^{0}\left(E, N_{E / \mathbb{P}^{r}}(-S)\right)$, one has $\operatorname{dim} R=h^{0}\left(E, N_{E / \mathbb{P}^{r}}(-S)\right)=h^{0}\left(E, N_{E / \mathbb{P}^{r}}\right)-(r-1)(r+1)=\operatorname{dim}_{[E]} \operatorname{Hilb}_{1, r+1}^{r}-(r-$ 1) $(r+1)=(r+1)^{2}-\left(r^{2}-1\right)=2 r+2$. As a consequence

$$
\begin{aligned}
n & =\underbrace{3(g-r-1)-4+\left(\begin{array}{c}
r+1 \\
2
\end{array}\right)}_{\operatorname{dim} W_{g-r-1}^{r-1}}+\underbrace{r}_{\text {choice of } H \subset \mathbb{P}^{r}}+\underbrace{r+1}_{\text {choice of } S}+\underbrace{2 r+2}_{\operatorname{dim} R} \\
& =3 g-4+\left(\begin{array}{c}
r+2 \\
2
\end{array}\right)-1=\operatorname{dim} W_{g}^{r}-1 .
\end{aligned}
$$

\subsection{Nodal curves in $\mathbb{P}^{r}$ with limit subcanonical points}

Consider the integers $r \geq 2$ and $g=g(r):=\left(\begin{array}{c}r+2 \\ 2\end{array}\right)$. As we pointed out in the previous section, the general point of the component $\mathcal{V}_{g}^{r} \subset \mathcal{S}_{g}^{r}$ is a pair $[C, L]$, where $[C] \in W_{g \text {,sm }}^{r}$ and $L \cong \mathcal{O}_{C}(1)$. We are interested in proving the existence of curves $[C] \in W_{g \text {,sm }}^{r}$ which admit a point $p \in C$ satisfying $L \cong \mathcal{O}_{C}((g-1) p)$, so that $p$ is a subcanonical point of $C$ and 
$[C, p] \in \mathcal{G}_{g}^{r}$. We note that $L \cong \mathcal{O}_{C}((g-1) p)$ if and only if there exists a hyperplane $M \subset \mathbb{P}^{r}$ such that the multiplicity of the intersection between $C$ and $M$ at $p$ is $\operatorname{mult}_{p}(C, M)=g-1$. Then it is natural to extend in these terms the notion of subcanonical points to every curve in $W_{g}^{r}$. Namely,

Definition 3.4 For any curve $[C] \in W_{g}^{r}$, we say that $p \in C$ is a limit subcanonical point if there exists a hyperplane $M \subset \mathbb{P}^{r}$ such that $M$ does not contain any component of $C$, and it cuts out on $C$ a 0 -dimensional scheme of length $g-1$ supported at a $p$.

We denote by

$$
Q_{g}^{r}:=\left\{[C] \in W_{g}^{r} \mid \exists M \subset \mathbb{P}^{r} \text { hyperplane, } \exists p \in M \text { s.t. } \operatorname{mult}_{p}(C, M)=g-1\right\}
$$

the sublocus of curves admitting a limit subcanonical point, and we set $Q_{g, \mathrm{sm}}^{r}:=Q_{g}^{r} \cap$ $W_{g, \mathrm{sm}}^{r}$ and $Q_{g, h}^{r}:=Q_{g}^{r} \cap W_{g, h}^{r}$. In particular, $Q_{g}^{r}$ is naturally endowed with the structure of subvariety of $W_{g}^{r}$. Analogously, fixing a hyperplane $M \subset \mathbb{P}^{r}$, we can define the sublocus of $Q_{g}^{r}(M) \subset Q_{g}^{r}$ of curves possessing a limit subcanonical point cut out by $M$, together with $Q_{g, \mathrm{sm}}^{r}(M):=Q_{g}^{r}(M) \cap W_{g, \mathrm{sm}}^{r}$ and $Q_{g, h}^{r}(M):=Q_{g}^{r}(M) \cap W_{g, h}^{r}$.

The reducible curves presented in the following remark provide an important class of nodal curves admitting a limit subcanonical point, which describe the locus $Q_{g, h}^{r}$ and play a crucial role in the proof of Theorem 1.2.

Remark 3.5 Let $[X] \in Q_{g, h}^{r} \subset W_{g, h}^{r}$ be a general point. Hence $X=C \cup E \subset \mathbb{P}^{r}$ as in Sect. 3.1, and there exists a hyperplane $M \subset \mathbb{P}^{r}$ and a point $p \in M$ such that $\operatorname{mult}_{p}(X, M)=$ $g(r)-1$. Since $\operatorname{deg} C=g(r-1)-1$ and $\operatorname{deg} E=g(r)-g(r-1)=r+1$, we have that $p \in C \cap E{\text {, } \text { mult }_{p}(C, M)=g(r-1)-1 \text { and mult }}_{p}(E, M)=r+1$. In particular, the curve $C \subset H \cong \mathbb{P}^{r-1}$ is such that $[C] \in Q_{g(r-1) \text {,sm }}^{r-1}$, and the subcanonical point $p \in C$ is cut out by $H \cap M$. Besides, the elliptic normal curve $E \subset \mathbb{P}^{r}$ has an inflection point of order $r+1$ at $p$, whose osculating plane is $M$.

Conversely, if $[X] \in W_{g, h}^{r}$ parameterizes a curve $X=C \cup E$ as in Sect. 3.1 such that the

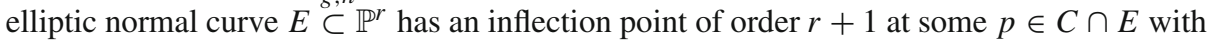
osculating hyperplane $M \subset \mathbb{P}^{r}$, and the smooth curve $C \subset H \cong \mathbb{P}^{r-1}$ has a subcanonical point at $p$ cut out by $H \cap M$, then $X$ admits a limit subcanonical point at $p$ cut out by $M$, so that $[X] \in Q_{g, h}^{r}$.

Remark 3.6 We recall that any curve $[C] \in Q_{g}^{r}(M)$ has degree $g-1$, and hence there exists a unique limit subcanonical point on $C$ which is cut out by $M$. Thus, for any hyperplane $M \subset$ $\mathbb{P}^{r}$, there is a surjective morphism $f: Q_{g}^{r}(M) \longrightarrow M$, whose fibres $Q_{g}^{r}(M, p):=f^{-1}(p)$ are described by the curves $[C] \in Q_{g}^{r}(M)$ such that mult $p(C, M)=g-1$. In addition, for any $p, q \in M$, there are isomorphisms $Q_{g}^{r}(M, p) \cong Q_{g}^{r}(M, q)$ induced by projectivities $\tau \in \operatorname{PGL}(r+1)$ such that $\tau(M)=M$ and $\tau(p)=q$.

We point out that for any hyperplane $M \subset \mathbb{P}^{r}$, there exists a natural modular map

$$
\begin{aligned}
\mu: Q_{g, \mathrm{sm}}^{r}(M) & \longrightarrow \mathcal{G}_{g}^{r} \subset \mathcal{M}_{g, 1} \\
{[C] } & \longmapsto[C, p]
\end{aligned}
$$

where $p \in C$ is the subcanonical point cut out by $M$. Then the following holds.

Lemma 3.7 Let $M \subset \mathbb{P}^{r}$ be a hyperplane, and let $Z \subset Q_{g, \mathrm{sm}}^{r}(M)$ be an irreducible component, whose general point $[C] \in Z$ parameterizes a non-degenerate linearly normal curve

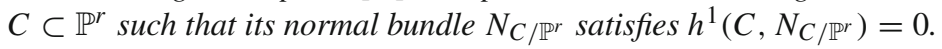


Then $Z$ dominates under $\mu$ an irreducible component $\mathcal{Z} \subset \mathcal{G}_{g}^{r}$ of dimension

$$
\operatorname{dim} \mathcal{Z}=\operatorname{dim} Z-\left[(r+1)^{2}-1-r\right] .
$$

Proof Let $[C] \in Z$ be a general point, and let $p \in C$ be the subcanonical point cut out by $M$, so that $\mu([C])=[C, p]$. Since $h^{1}\left(C, N_{C / \mathbb{P}^{r}}\right)=0$, the point $[C] \in \mathrm{Hilb}_{g, g-1}^{r}$ is smooth, hence there exists a unique irreducible component of $\mathrm{Hilb}_{g, g-1}^{r}$ passing through [C], that is $W_{g}^{r}$. Moreover, being $C \subset \mathbb{P}^{r}$ a smooth linearly normal curve, we deduce that $\mathcal{O}_{C}(1) \cong \mathcal{O}_{C}((g-1) p)$ is a very ample line bundle, with $h^{0}\left(C, \mathcal{O}_{C}((g-1) p)\right)=r+1$. In particular, if $C^{\prime} \subset \mathbb{P}^{r}$ is a curve such that $\left[C^{\prime}, p^{\prime}\right]=[C, p] \in \mathcal{Z}$ for some $p^{\prime} \in C^{\prime}$, then $C^{\prime}$ is projectively equivalent to $C$, so that $\left[C^{\prime}\right] \in W_{g}^{r}$.

Therefore, given an irreducible component $\mathcal{Z} \subset \mathcal{G}_{g}^{r}$ containing $\mu(Z)$ and an open neighbourhood $U \subset \mathcal{Z}$ of $[C, p]$, there exist a base change $U^{\prime} \longrightarrow U$ and a family of pointed curves $\left(\mathcal{C} \longrightarrow U^{\prime}, \rho: U^{\prime} \longrightarrow \mathcal{C}\right)$ such that any fibre $C_{t} \subset \mathbb{P}^{r}$ is a linearly normal curve having $\mathcal{O}_{C_{t}}(1) \cong \mathcal{O}_{C_{t}}((g-1) \rho(t))$. Since this family specializes to a pair $\left(C^{\prime}, p^{\prime}\right) \cong(C, p)$ and since $[C] \in \operatorname{Hilb}_{g, g-1}^{r}$ is a smooth point, we conclude that $U^{\prime} \subset Z$, so that $\mu_{\mid Z}: Z \longrightarrow \mathcal{Z}$ is dominant.

Finally, since the number of subcanonical points on a smooth curve is finite, the fibre of $\mu$ at some $[C, p] \in \operatorname{Im}(\mu)$ has the same dimension of the space of projectivities in $\operatorname{PGL}(r+1)$ sending $M$ to itself, that is $(r+1)^{2}-1-r$. Thus $\operatorname{dim} \mathcal{Z}=\operatorname{dim} \mu(Z)=$ $\operatorname{dim} Z-\left[(r+1)^{2}-1-r\right]$, as claimed.

Along the lines of Remark 3.5, we present a criterion for constructing inductively components of $Q_{g(r+1), h}^{r+1}$ by means of irreducible components of $Q_{g(r), \mathrm{sm}}^{r}$.

Lemma 3.8 Let $r \geq 2$ and $g(r)=\left(\begin{array}{c}r+2 \\ 2\end{array}\right)$. Consider a hyperplane $M \subset \mathbb{P}^{r}$, and let $Z \subset$ $Q_{g(r), \mathrm{sm}}^{r}(M)$ be an irreducible component of dimension $2 g(r)-2-\frac{r(r-1)}{2}+(r+1)^{2}-r$, whose general point $[C] \in Z$ parameterizes a non-degenerate linearly normal curve $C \subset \mathbb{P}^{r}$ such that $h^{1}\left(C, N_{C / \mathbb{P}^{r}}\right)=0$.

Then, for any hyperplane $M^{\prime} \subset \mathbb{P}^{r+1}$, there exists an irreducible component $B \subset$ $Q_{g(r+1), h}^{r+1}\left(M^{\prime}\right)$ of dimension $2 g(r+1)-4-\frac{r(r+1)}{2}+(r+2)^{2}-r$. Moreover, the general point $[X] \in B$ parameterizes a non-degenerate linearly normal curve $X \subset \mathbb{P}^{r+1}$ such that $h^{1}\left(X, N_{X / \mathbb{P}^{r+1}}\right)=0$.

Proof Let $H, M^{\prime} \subset \mathbb{P}^{r+1}$ be distinct hyperplanes and let $p^{\prime} \in H \cap M^{\prime}$. Let $[C] \in Z$ be as above, and let $p \in C$ be the point such that $\operatorname{mult}_{p}(C, M)=g(r)-1$. Let us consider an isomorphism $\iota_{H, p^{\prime}}: \mathbb{P}^{r} \longrightarrow H$ such that $\iota_{H, p^{\prime}}(p)=p^{\prime}$ and the $(r-1)$-plane $M \subset \mathbb{P}^{r}$ maps to $H \cap M^{\prime}$. So we identify $p, C$ and $M$ with their images in $H \subset \mathbb{P}^{r+1}$.

Let $Z_{p}:=Z \cap Q_{g}^{r}(M, p)$ be the sublocus of $Z$ described by curves having a subcanonical point at $p \in M$, and notice that it is an irreducible component of $Q_{g}^{r}(M, p) \cap W_{g, \mathrm{sm}}^{r}$ having dimension $\operatorname{dim} Z_{p}=\operatorname{dim} Z-(r-1)$ (cf. Remark 3.6). Let $C^{(r+2)}$ be the $(r+2)$-fold symmetric product of $C$, and consider the codimension one subvariety $V_{p}:=$ $\left\{p+D \in C^{(r+2)} \mid D \in C^{(r+1)}\right\}$ of effective divisors of degree $r+2$ on $C \subset \mathbb{P}^{r}$ containing $p$. We define $R_{V_{p}} \subset$ Hilb $_{1, r+2}^{r+1}$ as the subscheme parameterizing smooth elliptic normal curves $E$ of degree $r+2$, for which there exists a smooth divisor $S_{p} \in V_{p}$ such that $\operatorname{Supp}\left(S_{p}\right) \subset E$ and $\operatorname{mult}_{p}\left(E, M^{\prime}\right)=r+2$. From the fact that $E$ is smooth, it is easy to deduce that the points of $\operatorname{Supp}\left(S_{p}\right)$ are in linearly general position in $\mathbb{P}^{r}$.

Claim 3.9 Any irreducible component $R$ of $R_{V_{p}} \subset \mathrm{Hilb}_{1, r+2}^{r+1}$ has dimension $2 r+4$. 
Proof of Claim 3.9 Fix a general divisor $S_{p} \in V_{p}$, and let $R^{\prime} \subset \mathrm{Hilb}_{1, r+2}^{r+1}$ be the subscheme parameterizing smooth elliptic normal curves $E$ of degree $r+2$, with mult $_{p}\left(E, M^{\prime}\right)=$ $r+2$. We point out that any such a curve may be constructed by taking a smooth elliptic curve $E$ and a point $q \in E$, so that the complete linear system $\left|\mathcal{O}_{E}((r+2) q)\right|$ defines an embedding $\varphi_{\left|\mathcal{O}_{E}((r+2) q)\right|}: E \hookrightarrow \mathbb{P}^{r+1}$, which may be chosen such that $\varphi_{\left|\mathcal{O}_{E}((r+2) q)\right|}(q)=p$ and mult ${ }_{p}\left(E, M^{\prime}\right)=r+2$. Hence $R^{\prime}$ is irreducible, because there is a fibration $R^{\prime} \longrightarrow \mathcal{M}_{1,1}$ over the moduli space $\mathcal{M}_{1,1}$ of pointed elliptic curves given by $[E] \mapsto[E, p]$, and the fibre is isomorphic to the space $P$ of projectivities of $\mathbb{P}^{r+1}$ fixing $p$ and sending $M^{\prime}$ in itself. In particular, the dimension of $P$ is $\operatorname{dim} P=\operatorname{dim} \operatorname{PGL}(r+2)-(2 r+1)=r^{2}+2 r+2$ : it suffices to choose coordinates $x_{0}, \ldots, x_{r+1}$ on $\mathbb{P}^{r+1}$, set $p=[0: \ldots: 0: 1]$ and $M^{\prime}=\left\{x_{0}=0\right\}$, and notice that the space at hand is represented by matrices $\left[a_{i, j}\right]_{0 \leq i, j \leq r+1}$ such that $a_{1, l}=0$ for $1 \leq l \leq r+1$, and $a_{m, r+1}=0$ for $0 \leq m \leq r$. It follows that $\operatorname{dim} R^{\prime}=\operatorname{dim} \mathcal{M}_{1,1}+\operatorname{dim} P=r^{2}+2 r+3$.

Let $T \subset \mathbb{P}^{r+1}$ be a general hyperplane through $p$ and let $E \cap T=\left\{p, q_{1}, \ldots, q_{r+1}\right\}$, so that $(E \cap T) \backslash p$ consists of points of $\mathbb{P}^{r+1}$ in linearly general position. We note that the set $\operatorname{Supp}\left(S_{p}\right) \backslash p \subset \mathbb{P}^{r+1}$ consists of points in linearly general position, as well. As $\operatorname{dim} P>(r+1)(r+1)$, there exists some projective transformation in $P$ sending $(E \cap T) \backslash p$ to $\operatorname{Supp}\left(S_{p}\right) \backslash p$. Thus there exists a smooth curve $E$ parameterized over $R^{\prime}$ and passing through $\operatorname{Supp}\left(S_{p}\right)$. In particular, $E$ intersects the curve $C$ transversally at $S_{p}$. Moreover, since the points of $\operatorname{Supp}\left(S_{p}\right) \backslash p \subset \mathbb{P}^{r+1}$ are projectively equivalent to $r+1$ general points of $\mathbb{P}^{r+1}$, they impose exactly $r(r+1)=r^{2}+r$ conditions to curves of $R^{\prime}$. Therefore, if $R_{S_{p}} \subset R^{\prime}$ is the subscheme of curves passing through $\operatorname{Supp}\left(S_{p}\right)$, and $R^{\prime \prime} \subset R_{S_{p}}$ is an irreducible component containing [E], we deduce $\operatorname{dim} R^{\prime \prime}=\operatorname{dim} R^{\prime}-\left(r^{2}+r\right)=r+3$.

Finally, we let $S_{p}$ vary in $V_{p}$, and we consider an irreducible component $R$ of $R_{V_{p}}$ containing $R^{\prime \prime}$. Since $R$ is fibred over $V_{p}$, we conclude that $\operatorname{dim} R=\operatorname{dim} R^{\prime \prime}+\operatorname{dim} V_{p}=2 r+4$.

We recall that for any $[E] \in R_{V_{p}}$, the elliptic curve $E$ meets $C$ transversally at a general divisor $S_{p} \in V_{p}$, so that the reducible curve $X:=C \cup E$ has arithmetic genus $g(r)+$ $r+2=g(r+1)$. Furthermore, under the identification of $p \in C$ and $p^{\prime} \in M^{\prime}$, we have mult $_{p^{\prime}}\left(X, M^{\prime}\right)=$ mult $_{p^{\prime}}\left(C, M^{\prime}\right)+$ mult $_{p^{\prime}}\left(E, M^{\prime}\right)=g+r+1=g(r+1)-1$, and hence $[X] \in Q_{g(r+1), h}^{r+1}\left(M^{\prime}\right)$. We shall construct the irreducible component $B \subset Q_{g(r+1), h}^{r+1}\left(M^{\prime}\right)$, by letting vary $[C] \in Z_{p}$, the hyperplane $H \subset \mathbb{P}^{r+1},[E] \in R_{V_{p^{\prime}}}$ and $p^{\prime} \in M^{\prime}$.

Let $Z_{p}^{\circ} \subset Z_{p}$ be the open subset parameterizing non-degenerate curves. Consider the incidence variety $\mathcal{I}:=\left\{\left(p^{\prime},[H]\right) \in M^{\prime} \times\left(\left(\mathbb{P}^{r+1}\right)^{*} \backslash\left[M^{\prime}\right]\right) \mid p^{\prime} \in H \cap M^{\prime}\right\}$, and let $\iota: \mathbb{P}^{r} \times \mathcal{I} \longrightarrow \mathbb{P}^{r+1}$ be a morphism such that for any pair $\left(p^{\prime},[H]\right) \in \mathcal{I}$, the map $\iota\left(\cdot,\left(p^{\prime},[H]\right)\right): \mathbb{P}^{r} \hookrightarrow H$ is an inclusion, with $\iota\left(p,\left(p^{\prime},[H]\right)\right)=p^{\prime}$ and $\iota\left(M,\left(p^{\prime},[H]\right)\right)=H \cap M^{\prime}$. We point out that $\iota$ induces an inclusion $Z_{p}^{\circ} \times \mathcal{I} \hookrightarrow \mathrm{Hilb}_{g, g-1}^{r+1}$, which sends a pair $\left([\Gamma],\left(p^{\prime},[H]\right)\right)$ to the point $\left[\iota\left(\Gamma,\left(p^{\prime},[H]\right)\right)\right]$ parameterizing the curve $\Gamma$ embedded into $H$. We denote by $Z^{\prime} \subset$ Hilb $_{g, g-1}^{r+1}$ the image of $Z_{p}^{\circ} \times \mathcal{I}$, and let $\alpha_{2}: Z^{\prime} \longrightarrow \mathcal{I}$ be the second projection. In particular, $Z^{\prime}$ is an irreducible subvariety of dimension $\operatorname{dim} Z_{p}+\operatorname{dim} \mathcal{I}=(\operatorname{dim} Z-(r-1))+2 r=\operatorname{dim} Z+r+1$.

We consider the universal family $\mathcal{C} \subset \mathbb{P}^{r+1} \times Z^{\prime} \stackrel{\psi}{\rightarrow} Z^{\prime}$, the relative hyperplane bundle $\mathcal{L}:=\mathcal{O}_{\mathcal{C}}(1)$, and the $(r+2)$-fold relative symmetric product $\mathcal{C}^{(r+2)} \stackrel{\Psi}{\rightarrow} Z^{\prime}$ of $\psi$. Moreover, we define a divisor $\mathcal{D} \subset \mathcal{C}^{(r+2)}$ as

$$
\mathcal{D}:=\left\{p^{\prime \prime}+D \in \Gamma^{(r+2)} \mid[\Gamma] \in Z^{\prime}, \alpha_{2}[\Gamma]=\left(p^{\prime \prime},[H]\right) \text { and } D \in \Gamma^{(r+1)}\right\},
$$


whose restriction to any fibre $\Gamma$ of $\psi$ parameterizes the effective divisors on $\Gamma$ having degree $r+2$ and containing the corresponding $p^{\prime \prime}$. Clearly one has $\operatorname{dim} \mathcal{D}=r+1+\operatorname{dim} Z^{\prime}$. Let $\gamma_{2}: \mathbb{P}^{r+1} \times \mathcal{D} \rightarrow \mathcal{D}$ be the projection on the second factor, and let Hilb $\mathrm{b}_{1, r+2}\left(\gamma_{2}\right)$ be the relative Hilbert scheme of $\gamma_{2}$ parameterizing curves of arithmetic genus 1 and degree $r+2$ contained in the fibres of $\gamma_{2}$. Let $\mathcal{E} \subset$ Hilb $_{1, r+2}\left(\gamma_{2}\right)$ denote the subscheme described by non-degenerate smooth curves, and let $\varepsilon: \mathcal{E} \longrightarrow \mathcal{D}$ be the natural map inherited from Hilb $\mathrm{H}_{1, r+2}\left(\gamma_{2}\right)$. Then we consider the subvariety of $\mathcal{E}$ given by

$$
\mathcal{Q}:=\left\{\begin{array}{l|l}
{[E] \in \mathcal{E}} & \begin{array}{l}
\operatorname{mult}_{p^{\prime \prime}}\left(E, M^{\prime}\right)=r+2 \text { and }\left\langle p^{\prime \prime}+D\right\rangle \cap E=p^{\prime \prime}+D \\
\text { for } \varepsilon([E])=p^{\prime \prime}+D
\end{array}
\end{array}\right\},
$$

where $\langle\cdot\rangle$ denotes the linear span in $\mathbb{P}^{r}$. Since $V_{p^{\prime}} \subset \mathcal{D}$ by construction, for any irreducible component $R \subset R_{V_{p^{\prime}}}$ as in Claim 3.9, we have that any curve [E] $\in R$ lies on $\mathcal{Q}$. Conversely, if $[E] \in \mathcal{Q}$ is such that $\varepsilon([E])=S_{p^{\prime}}$ with $S_{p^{\prime}} \in V_{p^{\prime}}$ general, then $[E] \in R_{V_{p^{\prime}}}$. So we fix an irreducible component $R \subset R_{V_{p^{\prime}}}$, and we consider an irreducible component $\mathcal{B} \subset \mathcal{Q}$ containing $R$. Therefore $\mathcal{B}$ is fibred over the irreducible scheme $Z^{\prime}$ via the composition map

$$
\xi: \mathcal{B} \stackrel{\varepsilon}{\rightarrow} \mathcal{D} \stackrel{\Psi}{\rightarrow} Z^{\prime}
$$

The fibre over a general point $\left[C^{\prime}\right] \in Z^{\prime}$, with $\alpha_{2}\left[C^{\prime}\right]=\left(p^{\prime},[H]\right)$, is an irreducible component of the scheme $R_{V_{p^{\prime}}}$, which has dimension $2 r+4$ by Claim 3.9. Thus

$$
\begin{aligned}
\operatorname{dim} \mathcal{B} & =\operatorname{dim} Z^{\prime}+\operatorname{dim} R_{V_{p^{\prime}}}=\operatorname{dim} Z+r+1+2 r+4 \\
& =\left[2 g(r)-2-\frac{r(r-1)}{2}+(r+1)^{2}-r\right]+r+1+2 r+4 \\
& =2 g(r+1)-4-\frac{r(r+1)}{2}+(r+2)^{2}-r .
\end{aligned}
$$

Finally, we point out that $\mathcal{B}$ is isomorphic to the scheme

$$
B^{\circ}:=\left\{[C \cup E] \in W_{g(r+1), h}^{r+1} \mid[C] \in Z^{\prime} \text { and }[E] \in \xi^{-1}([C])\right\} .
$$

We recall that $Z$ is an irreducible component of $Q_{g, \mathrm{sm}}^{r}(M)$, and $\mathcal{D}$ is irreducible. Then it is straightforward to check that the Zariski closure of $B^{\circ}$ must describe a whole irreducible component $B$ of $Q_{g(r+1), h}^{r+1}\left(M^{\prime}\right)$.

For the last part of the statement, let $[X] \in B$ be a general point, with $X:=C \cup E$, $\alpha_{2}[C]=\left(p^{\prime},[H]\right)$, and $\varepsilon([E])=S_{p^{\prime}}$. In order to show that $X$ is linearly normal, it suffices to compute the cohomology of the Mayer-Vietoris sequence

$$
0 \rightarrow \mathcal{I}_{X / \mathbb{P}^{r+1}}(1) \rightarrow \mathcal{I}_{C / \mathbb{P}^{r+1}}(1) \oplus \mathcal{I}_{E / \mathbb{P}^{r+1}}(1) \rightarrow \mathcal{I}_{S_{p^{\prime}} / \mathbb{P}^{r+1}}(1) \rightarrow 0 .
$$

Since $C \subset H$ is linearly normal by assumption, one has $h^{1}\left(\mathcal{I}_{C / H}(1)\right)=0$. Using the exact sequence $0 \rightarrow \mathcal{I}_{H / \mathbb{P}^{r+1}}(1) \cong \mathcal{O}_{\mathbb{P}^{r+1}} \rightarrow \mathcal{I}_{C / \mathbb{P}^{r+1}}(1) \rightarrow \mathcal{I}_{C / H}(1) \rightarrow 0$, we immediately deduce $h^{1}\left(\mathcal{I}_{C / \mathbb{P}^{r+1}}(1)\right)=0$. On the other hand, $E \subset \mathbb{P}^{r+1}$ is linearly normal, hence $h^{1}\left(\mathcal{I}_{E / \mathbb{P}^{r+1}}(1)\right)=0$. Since $\langle C\rangle=H \cong \mathbb{P}^{r},\langle E\rangle=\mathbb{P}^{r+1}$ and $\left\langle S_{p^{\prime}}\right\rangle=H$, we have $h^{0}\left(\mathcal{I}_{C / \mathbb{P}^{r+1}}(1)\right)=1, h^{0}\left(\mathcal{I}_{E / \mathbb{P}^{r+1}}(1)\right)=0$ and $h^{0}\left(\mathcal{I}_{S_{p^{\prime}} / \mathbb{P}^{r+1}}(1)\right)=1$. Furthermore, $X \subset \mathbb{P}^{r+1}$ is non-degenerate, and hence $h^{0}\left(\mathcal{I}_{X / \mathbb{P}^{r+1}}(1)\right)=0$. Therefore $h^{1}\left(\mathcal{I}_{X / \mathbb{P}^{r+1}}(1)\right)=0$, that is $X$ is linearly normal.

To conclude, assume that $h^{1}\left(C, N_{C / \mathbb{P}^{r}}\right)=0$. Since $S_{p^{\prime}} \in V_{p^{\prime}}$ is general, the $r+1$ points of $\operatorname{Supp}\left(S_{p^{\prime}}\right) \backslash p^{\prime}$ are general as points of $C$. Thus, it follows immediately from the geometric version of Riemann-Roch Theorem that $h^{1}\left(C, \mathcal{O}_{C}\left(\Sigma+S_{p^{\prime}}\right)\right)=0$, where $\Sigma$ is a hyperplane 
section of $C$. Then the proof of the fact that $h^{1}\left(X, N_{X / \mathbb{P}^{r+1}}\right)=0$ goes on exactly as in the proof of [4, Lemma 3.2].

\subsection{Components of $\mathcal{G}_{g(r)}^{r}$ having expected dimension}

We are going to prove that any locus $\mathcal{G}_{g(r)}^{r} \subset \mathcal{M}_{g(r), 1}$ of subcanonical points admits an irreducible component $\mathcal{Z}_{g(r)}$ of expected dimension. In analogy with [5], we shall construct a component $Z_{g(r)} \subset Q_{g(r) \text {,sm }}^{r}$ of suitable dimension in the Hilbert scheme of curves in $\mathbb{P}^{r}$, and we shall map it in the moduli space $\mathcal{M}_{g(r), 1}$ to obtain the component $\mathcal{Z}_{g(r)} \subset \mathcal{G}_{g(r)}^{r}$.

Theorem 3.10 For any $r \geq 2$ and $g(r)=\left(\begin{array}{c}r+2 \\ 2\end{array}\right)$, there exists an irreducible component $\mathcal{Z}_{g(r)}$ of $\mathcal{G}_{g(r)}^{r}$ having expected dimension $2 g(r)-1-\frac{r(r-1)}{2}$, and such that its general point $[C, p] \in \mathcal{Z}_{g(r)}$ satisfies $h^{0}\left(C, O_{C}((g(r)-1) p)\right)=r+1$.

Proof We want to prove that for any $r \geq 2$ and for any hyperplane $M \subset \mathbb{P}^{r}$, there exists an irreducible component $Z_{g(r)} \subset Q_{g(r), \mathrm{sm}}^{r}(M)$ such that $\operatorname{dim} Z_{g(r)}=2 g(r)-2-\frac{r(r-1)}{2}+$ $(r+1)^{2}-r$, and the general point $[C] \in Z_{g(r)}$ parameterizes a non-degenerate linearly normal curve $C \subset \mathbb{P}^{r}$ with $h^{1}\left(C, N_{C / \mathbb{P}^{r}}\right)=0$.

Indeed, if such a component exists, Lemma 3.7 assures that the image of $Z_{g(r)}$ under the modular map $\mu: Q_{g(r), \mathrm{sm}}^{r}(M) \longrightarrow \mathcal{M}_{g(r), 1}$ is an irreducible component $\mathcal{Z}_{g(r)} \subset \mathcal{G}_{g(r)}^{r}$ having dimension $\operatorname{dim} \mathcal{Z}_{g(r)}=\operatorname{dim} Z_{g(r)}-\left[(r+1)^{2}-1-r\right]=2 g(r)-1-\frac{r(r-1)}{2}$. Moreover, as the general point $[C] \in Z_{g(r)}$ parameterizes a non-degenerate linearly normal curve $C \subset \mathbb{P}^{r}$ admitting a divisor $(g(r)-1) p$ cut out by a hyperplane $M \subset \mathbb{P}^{r}$, we deduce that $h^{0}\left(C, \mathcal{O}_{C}((g(r)-1) p)\right)=h^{0}\left(C, \mathcal{O}_{C}(1)\right)=r+1$, so that the assertion follows.

We point out that when $r=2$, such a component $Z_{6}=Z_{g(2)}$ does exist. Given any line $M \subset \mathbb{P}^{2}$, we define $Z_{6} \subset$ Hilb $_{6,5}^{2}$ as the locus of smooth quintic curves $C \subset \mathbb{P}^{2}$ such that $M$ meets $C$ at a single point $p \in C$, as in Example 2.6. We recall that pairs $[C, p] \in \mathcal{M}_{6,1}$ as above describe an irreducible component $\mathcal{Z}_{6} \subset \mathcal{G}_{6}^{2}$ having dimension $2 g(r)-1-\frac{r(r-1)}{2}=10$. Therefore, it is easy to see that $\operatorname{dim} Z_{6}=\operatorname{dim} \mathcal{Z}_{6}+(r+1)^{2}-1-r=16$ (cf. Lemma 3.7). We note further that the general curve $[C] \in Z_{6}$ parameterizes a linearly normal curve, as $\mathcal{O}_{C}(5 p) \cong \mathcal{O}_{C}(1)$ possesses exactly 3 independent global sections (see Example 2.6), and that $h^{1}\left(C, N_{C / \mathbb{P}^{2}}\right)=0$ as $N_{C / \mathbb{P}^{2}} \cong \mathcal{O}_{C}(C)$.

Then we argue by induction on $r$, and we want to prove that for any hyperplane $M^{\prime} \subset \mathbb{P}^{r+1}$, there exists an irreducible component $Z_{g(r+1)} \subset Q_{g(r+1) \text {,sm }}^{r+1}\left(M^{\prime}\right)$ such that $\operatorname{dim} Z_{g(r+1)}=$ $2 g(r+1)-2-\frac{r(r+1)}{2}+(r+2)^{2}-r-1$, and the general point $[\Gamma] \in Z_{g(r+1)}$ parameterizes a non-degenerate linearly normal curve $\Gamma \subset \mathbb{P}^{r+1}$ with $h^{1}\left(\Gamma, N_{\Gamma / \mathbb{P}^{r+1}}\right)=0$. For the sake of simplicity, we write hereafter $g:=g(r+1)$.

Thanks to the inductive assumption, Lemma 3.8 assures that for any hyperplane $M^{\prime} \subset$ $\mathbb{P}^{r+1}$, there exists an irreducible component of $B \subset Q_{g, h}^{r+1}\left(M^{\prime}\right)$ having dimension $2 g-2-$ $\frac{r(r+1)}{2}+(r+2)^{2}-r-2$. We focus on the partial compactification of $W_{g \text {,sm }}^{r+1}$ given by $W_{g, \mathrm{sm}}^{r+1} \cup W_{g, h}^{r+1}$. Then we consider a nodal curve $X_{0}=C \cup E$ parameterized by a general point of $0=\left[X_{0}\right] \in B$, endowed with a limit subcanonical point $p^{\prime} \in X_{0}$ cut out by $M^{\prime}$, i.e. mult $_{p^{\prime}}\left(X_{0}, M^{\prime}\right)=g-1$. We are firstly aimed at bounding the dimension of any irreducible component of $Q_{g}^{r+1}\left(M^{\prime}\right)$ passing through the point $0 \in W_{g, \mathrm{sm}}^{r+1} \cup W_{g, h}^{r+1}$.

Let $\mathcal{X} \subset \mathbb{P}^{r+1} \times\left(W_{g, \mathrm{sm}}^{r+1} \cup W_{g, h}^{r+1}\right) \stackrel{\psi}{\longrightarrow}\left(W_{g, \mathrm{sm}}^{r+1} \cup W_{g, h}^{r+1}\right) \subset$ Hilb $_{g, g-1}^{r+1}$ be the universal family, and let $T \subset W_{g, \mathrm{sm}}^{r+1} \cup W_{g, h}^{r+1}$ be an analytic neighbourhood centred at 0 , so that-up 
to shrinking it $-T$ is smooth of dimension $\operatorname{dim} T=\operatorname{dim} W_{g}^{r+1}=3 g-4+\left(\begin{array}{c}r+3 \\ 2\end{array}\right)$ because $h^{1}\left(X_{0}, N_{X_{0} / \mathbb{P}^{r+1}}\right)=0$ by Lemma 3.8. Let us still denote by $\mathcal{X} \subset \mathbb{P}^{r+1} \times T \stackrel{\psi}{\longrightarrow} T$ the pull-back to $T$ of the universal family, and let $X_{t}:=\psi^{-1}(t)$ be the fibre over $t \in T$. We point out that the total space $\mathcal{X}$ is smooth. Indeed, the smoothness of $T$ ensures that $\mathcal{X}$ is smooth away from the singularities of its fibres. Besides, since the general fibre of $\psi$ is smooth and $h^{1}\left(X_{0}, N_{X_{0} / \mathbb{P}^{r+1}}\right)=0$, we deduce that—up to shrinking $T$ further - the family $\mathcal{X} \stackrel{\psi}{\longrightarrow} T$ is (the pull-back over $T$ of) a versal deformation of the nodes of $X_{0}$, hence $\mathcal{X}$ is smooth everywhere.

Following the argument for proving Theorem 1.1, we consider the $(g-1)$-fold relative symmetric product $\mathcal{X}^{(g-1)} \stackrel{\Psi}{\longrightarrow} T$ of the family $\psi$, so that the fibre over each $t \in T$ is the variety $\Psi^{-1}(t)=X_{t}^{(g-1)}$. In this case $\mathcal{X}^{(g-1)}$ does not coincide with the relative Hilbert scheme $\mathcal{X}^{[g-1]} \stackrel{\widetilde{\Psi}}{\longrightarrow} T$, which parameterizes 0 -dimensional subschemes of $\mathcal{X}$ of length $g-1$ contained in the fibres of $\psi$. In particular, it follows from the Blowup Theorem of [26] and the analysis therein that $\mathcal{X}^{[g-1]}$ is a smooth variety of dimension $\operatorname{dim} T+g-1$ fitting in the commutative diagram

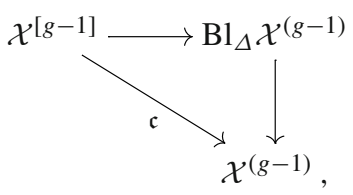

where $\mathfrak{c}$ is the cycle map given by $A \in \mathcal{X}^{[g-1]} \longmapsto \sum_{p \in \mathcal{X}} \operatorname{length}_{p}(A) p \in \mathcal{X}^{(g-1)}$, and $\mathrm{Bl}_{\Delta} \mathcal{X}^{(g-1)}$ is the blow up of $\mathcal{X}^{(g-1)}$ along the discriminant locus $\Delta$ described by non-reduced 0 -cycles.

Let $\widetilde{\widetilde{\Delta}}_{(g-1)} \subset \mathcal{X}^{[g-1]}$ be the locus parameterizing 0-dimensional schemes supported at a single point. By [25, Section 2.1], the locus $\widetilde{\Delta}_{(g-1)}$ has $\operatorname{dimension} \operatorname{dim} T+1$, and it maps birationally to the relative small diagonal $\Delta_{(g-1)}:=\left\{(g-1) q \in X_{t}^{(g-1)} \mid t \in T\right\} \subset \mathcal{X}^{(g-1)}$. Furthermore, the restriction $\mathfrak{c}_{\mid \tilde{\Delta}_{(g-1)}}: \tilde{\Delta}_{(g-1)} \longrightarrow \Delta_{(g-1)}$ is an isomorphism away from the nodes of the fibres of $\psi$, whereas if $q \in \operatorname{Sing}\left(X_{t}\right)$ for some $t \in T$, the preimage of $(g-1) q \in X_{t}^{(g-1)}$ consists of a chain of $g-2$ rational curves, which arises from the blow up in (3.1) (see also [23, Theorem 1]). In particular, considering the family $\widetilde{\Delta}_{(g-1)} \stackrel{\widetilde{\psi}}{\longrightarrow} T$ obtained from the morphism $\widetilde{\Psi}$, we have that if $X_{t}$ is a smooth curve, then the fibre $\widetilde{\psi}^{-1}(t)$ is isomorphic to the small diagonal $\left\{(g-1) q \in X_{t}^{(g-1)} \mid q \in X_{t}\right\}$ of $X_{t}^{(g-1)}$, which in turn is isomorphic to $X_{t}$. On the other hand, if the curve $X_{t}$ is singular, then $\widetilde{\psi}^{-1}(t)$ is a semistable model of $X_{t}$, where a chain of $g-2$ rational curves is inserted at any node of $X_{t}$. As for the family $\mathcal{X} \stackrel{\psi}{\longrightarrow} T$, we then deduce that $\widetilde{\Delta}_{(g-1)} \stackrel{\widetilde{\psi}}{\longrightarrow} T$ is a versal deformation of the nodes of $\widetilde{\psi}^{-1}(0)$, so that the total space $\widetilde{\Delta}_{(g-1)}$ turns out to be smooth.

Up to shrinking $T$, we may assume that the hyperplane $M^{\prime}$ has 0 -dimensional intersection with any curve parameterized by $T$, i.e. $M^{\prime}$ does not contain any irreducible component of the fibres $X_{t}$. So we can define the locus $\widetilde{\mathcal{Y}} \subset \mathcal{X}^{[g-1]}$ parameterizing 0 -cycles of length $g-1$ cut out by $M^{\prime}$ on the curves $X_{t}$, that is

$$
\tilde{\mathcal{Y}}:=\left\{\zeta \in X_{t}^{[g-1]} \mid t \in T \text { and } \zeta=\left.X_{t}\right|_{M^{\prime}}\right\}
$$


In particular, $\tilde{\mathcal{Y}}$ is the image of a regular section of the morphism $\mathcal{X}^{[g-1]} \stackrel{\widetilde{\Psi}}{\longrightarrow} T$, which assures that $\tilde{\mathcal{Y}}$ is smooth.

We note that a point $q \in X_{t}$ is a limit subcanonical point cut out by $M_{\tilde{\mathcal{V}}}^{\prime}$-i.e. $t=\left[X_{t}\right]$ lies on $Q_{g}^{r+1}\left(M^{\prime}, q\right)$-if and only if $\mathfrak{c}^{-1}((g-1) q) \in \tilde{\mathcal{Y}} \cap \widetilde{\Delta}_{(g-1)}$. Since $\widetilde{\mathcal{Y}}$ maps finitely to its image under $\widetilde{\Psi}$, also $\widetilde{\mathcal{Y}} \cap \widetilde{\Delta}_{(g-1)}$ does. Moreover, $0 \in \widetilde{\Psi}\left(\widetilde{\mathcal{Y}} \cap \widetilde{\Delta}_{(g-1)}\right) \subset T$ as $p^{\prime} \in X_{0}$ is a limit subcanonical point of $X_{0}$ cut out by $M^{\prime}$. Thus any irreducible component $Z^{\prime} \subset$ $Q_{g}^{r+1}\left(M^{\prime}\right)$ passing through 0 has dimension bounded by the least dimension of irreducible components of $\tilde{\mathcal{Y}} \cap \widetilde{\Delta}_{(g-1)}$. In particular, in view of the smoothness of $\mathcal{X}^{[g-1]}, \widetilde{\Delta}_{(g-1)}$ and $\tilde{\mathcal{Y}}$, we deduce

$$
\begin{aligned}
\operatorname{dim} Z^{\prime} & \geq \operatorname{dim} \tilde{\mathcal{Y}}+\operatorname{dim} \widetilde{\Delta}_{(g-1)}-\operatorname{dim} \mathcal{X}^{[g-1]} \\
& =\operatorname{dim} T+(\operatorname{dim} T+1)-(\operatorname{dim} T+g-1) \\
& =3 g-4+\left(\begin{array}{c}
r+3 \\
2
\end{array}\right)-g+2=2 g-2-\frac{r(r+1)}{2}+(r+2)^{2}-r-1 .
\end{aligned}
$$

Let us assume that $Z^{\prime} \subset Q_{g}^{r+1}\left(M^{\prime}\right) \cap\left(W_{g, \mathrm{sm}}^{r+1} \cup W_{g, h}^{r+1}\right)$ is an irreducible component containing $B$, and let $Z_{g} \subset Z^{\prime}$ be the-possibly empty-sublocus parameterizing smooth curves. We recall that $B \subset Q_{g, h}^{r+1}\left(M^{\prime}\right)$ is an irreducible component of the intersection $Z^{\prime} \cap W_{g, h}^{r+1}$, and $\operatorname{dim} B=2 g-2-\frac{r(r+1)}{2}+(r+2)^{2}-r-2$. By inequality (3.2) we have $\operatorname{dim} Z^{\prime} \geq \operatorname{dim} B+1$, so that $Z_{g} \subset Q_{g \text {,sm }}^{r+1}\left(M^{\prime}\right)$ is non-empty. Furthermore, Proposition 3.3 assures that $W_{g, h}^{r+1}$ is a divisorial component of $W_{g}^{r+1}$, and hence $\operatorname{dim} Z_{g}=\operatorname{dim} Z^{\prime}=$ $\operatorname{dim} B+1$.

Hence we achieved the existence of an irreducible component $Z_{g(r+1)}=Z_{g} \subset$ $Q_{g, \mathrm{sm}}^{r+1}\left(M^{\prime}\right)$ having the desired dimension. It remains to check that its general point $[\Gamma] \in Z_{g}$ parameterizes a linearly normal curve $\Gamma \subset \mathbb{P}^{r+1}$ with $h^{1}\left(\Gamma, N_{\Gamma / \mathbb{P}^{r+1}}\right)=0$. However, these facts follow easily as $B$ lies in the Zariski closure of $Z_{g} \subset \mathrm{Hilb}_{g, g-1}^{r+1}$, and the general point $[X] \in B$ parameterizes a linearly normal curve $X=C \cup E \subset \mathbb{P}^{r+1}$ with $h^{1}\left(X, N_{X / \mathbb{P}^{r+1}}\right)=0$.

\subsection{Proof of Theorem 1.2}

In the light of the previous analysis, proving the sharpness of Theorem 1.1 is now straightforward.

Proof of Theorem 1.2 As in the statement of the theorem, we consider integers $r \geq 0$ and $g \geq g(r)$, where $g(r)$ is given by (1.1), together with a partition $\underline{k}=\left(k_{1}, \ldots, k_{n}\right)$ of $g-1$. We are aimed at proving that the locus $\mathcal{G}_{g}^{r}(\underline{k})$ admits an irreducible component having expected dimension, whose general point $\left[C, p_{1}, \ldots, p_{n}\right]$ is such that $h^{0}\left(C, \mathcal{O}_{C}\left(\sum_{i=1}^{n} k_{i} p_{i}\right)\right)=r+1$ and-except for the cases $(r, g)=(0,2)$ and $(1,3)$ - the curve $C$ is non-hyperelliptic.

The case $2 \leq g \leq 3$ has been already studied in Example 2.1, and Theorem 1.2 holds under this assumption. Then we set hereafter $g \geq 4$, and we initially focus on the case $\underline{k}=(g-1)$. In the range $0 \leq r \leq 3$, the assertion of Theorem 1.2 on the locus $\mathcal{G}_{g}^{r}$ of subcanonical points is covered by Proposition 2.5.

Hence we assume $r \geq 4$, so that $g(r)=\left(\begin{array}{c}r+2 \\ 2\end{array}\right)$. By Theorems 3.10 and 2.7, for any $g \geq g(r)$ there exists an irreducible component of $\mathcal{Z}_{g} \subset \mathcal{G}_{g}^{r}$ having expected dimension $2 g-1-\frac{r(r-1)}{2}$, 
and such that $h^{0}\left(C, \mathcal{O}_{C}((g-1) p)\right)=r+1$ for general $[C, p] \in \mathcal{Z}_{g}$. In particular, the curve $C$ is non-hyperelliptic: we have $\operatorname{dim} \mathcal{Z}_{g} \leq 2 g-7$ for any $r \geq 4$, whereas hyperelliptic curves of genus $g$ just describe an irreducible component $\mathcal{G}_{g}^{\text {hyp }} \subset \mathcal{G}_{g}^{r}$ of dimension $2 g-1$, with $r \equiv\left\lfloor\frac{g-1}{2}\right\rfloor(\bmod 2)($ see Example 2.2 and Sect. 2.4)

Therefore Theorem 2.3 assures that for any partition $\underline{k}=\left(k_{1}, \ldots, k_{n}\right)$ of $g-1$, the locus $\mathcal{G}_{g}^{r}(\underline{k})$ admits an irreducible component $\mathcal{W}_{g}$ having expected dimension. If moreover $\left[D, q_{1}, \ldots, q_{n}\right] \in \mathcal{W}_{g}$ is a general point, then neither $D$ is hyperelliptic, nor $h^{0}\left(D, \mathcal{O}_{D}\left(\sum_{i=1}^{n} k_{i} q_{i}\right)\right)>r+1$.

Acknowledgements We would like to thank Enrico Arbarello, Andrea Bruno, Giulio Codogni, Donatella Iacono, Andreas Knutsen, Edoardo Sernesi, Alessandro Verra and Filippo Viviani for helpful discussions. We are also grateful to the anonymous referee for pointing out some inaccuracies and making valuable suggestions which improved this work.

\section{References}

1. Arbarello, E., Cornalba, M., Griffiths, P.A., Harris, J.: Geometry of Algebraic Curves, vol. I. Springer, New York (1985)

2. Ballico, E., Benzo, L., Fontanari, C.: Families of nodal curves in $\mathbb{P}^{r}$ with the expected number of moduli. Boll. Unione Mat. Ital. 7, 183-192 (2014)

3. Bastianelli, F., Pirola, G.P.: Subcanonical points on projective curves and triply periodic minimal surfaces in the Euclidean space. Math. Z. 279, 1029-1046 (2015)

4. Benzo, L.: Components of moduli spaces of spin curves with the expected codimension. Math. Ann. 363, 385-392 (2015)

5. Benzo, L.: Components of moduli spaces of spin curves with the expected codimension II (2015). arXiv: $1502.05262 \mathrm{v} 2$

6. Bullock, E.M.: Subcanonical points on algebraic curves. Trans. Am. Math. Soc. 365, 99-122 (2013)

7. Chen, D., Tarasca, N.: Loci of curves with subcanonical points in low genus. Math. Z. 284, 683-714 (2016)

8. Colombo, E.: On curves with a theta-characteristic whose space of sections has dimension 4. Math. Z. 215, 655-665 (1994)

9. Eisenbud, D., Harris, J.: Limit linear series: basic theory. Invent. Math. 85, 337-371 (1986)

10. Eisenbud, D., Harris, J.: When ramification points meet. Invent. Math. 87, 485-493 (1987)

11. Eisenbud, D., Harris, J.: Existence, decomposition, and limits of certain Weierstrass points. Invent. Math. 87, 495-515 (1987)

12. Farkas, G.: Gaussian maps, Gieseker-Petri loci and large theta-characteristics. J. Reine Angew. Math. 581, 151-173 (2005)

13. Farkas, G.: Theta characteristics and their moduli. Milan J. Math. 80, 1-24 (2012)

14. Farkas, G., Pandharipande, R.: The moduli space of twisted canonical divisors. J. Inst. Math. Jussieu (2016). https://doi.org/10.1017/S1474748016000128

15. Harris, J.: Theta-characteristics on algebraic curves. Trans. Am. Math. Soc. 271, 611-638 (1982)

16. Hartshorne, R.: Algebraic Geometry. Springer, Heidelberg (1977)

17. Kontsevich, M., Zorich, A.: Connected components of the moduli spaces of Abelian differentials with prescribed singularities. Invent. Math. 153, 631-678 (2003)

18. Mumford, D.: Theta-characteristic on an algebraic curve. Ann. Sci. École Norm. Sup. 4(4), 181-192 (1971)

19. Nagaraj, D.S.: On the moduli of curves with theta-characteristics. Compos. Math. 75, 287-297 (1990)

20. Pirola, G.P.: The infinitesimal variation of the spin abelian differentials and periodic minimal surfaces. Commun. Anal. Geom. 6, 393-426 (1998)

21. Polishchuk, A.: Moduli spaces of curves with effective $r$-spin structures. In: Jarvis, T.J., Kimura, T., Vaintrob, A. (eds.) Gromov-Witten Theory of Spin Curves and Orbifolds, pp. 1-20. American Mathematical Society, Providence (2006)

22. Ran, Z.: Geometry on nodal curves. Compos. Math. 141, 1191-1212 (2005)

23. Ran, Z.: A note on Hilbert schemes of nodal curves. J. Algebra 292, 429-446 (2005) 
24. Ran, Z.: Cycle map on Hilbert schemes of nodal curves. In: Ciliberto, C., Geramita, A.V., Harbourne, B., Miró-Roig, R.M., Ranestad, K. (eds.) Projective Varieties with Unexpected Properties, pp. 361-378. Walter de Gruyter GmbH \& Co. KG, Berlin (2005)

25. Ran, Z.: Tautological module and intersection theory on Hilbert schemes of nodal curves. Asian J. Math. 17, 193-263 (2013)

26. Ran, Z.: Structure of the cycle map for Hilbert schemes of families of nodal curves. Israel J. Math. 215, 669-711 (2016)

27. Teixidor i Bigas, M.: Half-canonical series on algebraic curves. Trans. Am. Math. Soc. 302, 99-115 (1987) 